\title{
VARIATION IN THE PRE-TRANSIT BALMER LINE SIGNAL AROUND THE HOT JUPITER HD 189733B
}

\author{
P. Wilson Cauley ${ }^{1}$, Seth Redfield ${ }^{1}$, Adam G. Jensen ${ }^{2}$, and Travis Barman ${ }^{3}$ \\ ${ }^{1}$ Wesleyan University Astronomy Department, Van Vleck Observatory, 96 Foss Hill Drive, Middletown, CT 06459, USA; pcauley@wesleyan.edu \\ ${ }^{2}$ University of Nebraska-Kearney Department of Physics \& Physical Science, 24011 11th Avenue, Kearney, NE 68849, USA \\ ${ }^{3}$ University of Arizona Department of Planetary Sciences and Lunar and Planetary Laboratory, 1629 E University Boulevard, Tucson, AZ 85721, USA \\ Received 2016 February 29; accepted 2016 May 3; published 2016 June 23
}

\begin{abstract}
As followup to our recent detection of a pre-transit signal around HD $189733 \mathrm{~b}$, we obtained full pre-transit phase coverage of a single planetary transit. The pre-transit signal is again detected in the Balmer lines but with variable strength and timing, suggesting that the bow shock geometry reported in our previous work does not describe the signal from the latest transit. We also demonstrate the use of the $\mathrm{Ca}$ II $\mathrm{H}$ and $\mathrm{K}$ residual core flux as a proxy for the stellar activity level throughout the transit. A moderate trend is found between the pre-transit absorption signal in the 2013 data and the Ca II H flux. This suggests that some of the 2013 pre-transit hydrogen absorption can be attributed to varying stellar activity levels. A very weak correlation is found between the $\mathrm{Ca}$ II $\mathrm{H}$ core flux and the Balmer line absorption in the 2015 transit, hinting at a smaller contribution from stellar activity compared to the 2013 transit. We simulate how varying stellar activity levels can produce changes in the Balmer line transmission spectra. These simulations show that the strength of the 2013 and 2015 pre-transit signals can be reproduced by stellar variability. If the pre-transit signature is attributed to circumplanetary material, its evolution in time can be described by accretion clumps spiraling toward the star, although this interpretation has serious limitations. Further high-cadence monitoring at $\mathrm{H} \alpha$ is necessary to distinguish between true absorption by transiting material and shortterm variations in the stellar activity level.
\end{abstract}

Key words: planets and satellites: atmospheres - planets and satellites: gaseous planets - planets and satellites: magnetic fields - stars: activity

\section{INTRODUCTION}

Hot exoplanets, i.e., exoplanets with orbital periods of approximately a few days, are unique laboratories for the study of star-planet interactions (hereafter SPIs). The large amounts of stellar UV and EUV radiation that these planets receive heats the planetary atmosphere and can result in significant photoevaporative mass loss across a broad range of planetary masses (e.g., Vidal-Madjar et al. 2003; Murray-Clay et al. 2009; Lecavelier des Etangs et al. 2010; Owen \& Jackson 2012; Lopez \& Fortney 2013; Ehrenreich et al. 2015). If the mass loss is significant and the planet overflows its Roche lobe, the outflowing planetary material can accrete onto the star (Lai et al. 2010; Matsakos et al. 2015; Pillitteri et al. 2015). The short orbital distances of these planets can also result in large tidal interactions, generating enhanced activity levels on the star that are directly related to the orbital phase of the planet (Cuntz et al. 2000; Shkolnik et al. 2008; Pillitteri et al. 2015). Direct magnetic interactions via reconnection events or magnetic torques are also possible and can affect the orbital and spin evolution of the planet and star (Lanza 2010; Cohen et al. 2010; Strugarek et al. 2014). Understanding these interactions is important in order to move toward a full characterization of exoplanets and a complete knowledge of their evolution under extreme conditions.

Two particularly interesting classes of SPIs are the direct interaction of escaping planetary material (i.e., the planetary wind) and the stellar wind and the interaction of the planet's magnetosphere with the stellar wind. Both scenarios are capable of producing bow shocks at the interaction region which results in a significant density enhancement of the shocked material (Vidotto et al. 2010; Bisikalo et al. 2013; Matsakos et al. 2015). For parameters relevant to most hot planet systems, the interaction region in both cases occurs ahead of the planet in its orbit. Observationally this can produce a pre-transit signature (for transiting or very nearly transiting planets) if the material ahead of the planet is sufficiently opaque to produce measurable absorption (Vidotto et al. 2010, 2011; Llama et al. 2011). In the case of a bow shock mediated by the planet's magnetosphere, it is possible to estimate the strength of the field given some assumptions about the stellar wind (Vidotto et al. 2010; Llama et al. 2013; Cauley et al. 2015). While estimates of this sort are rough, this method of measuring exoplanet magnetic fields may be worth pursuing considering the challenges of directly detecting emission from electrons in the planetary magnetosphere and the current lack of such confirmed detections (e.g., Murphy et al. 2015; Vidotto et al. 2015).

A third scenario capable of producing pre-transit absorption is the loss of planetary material that then accretes onto the central star. Accretion streams or time-variable blobs are predicted from some simulations of hot planet mass loss (Cohen et al. 2011; Matsakos et al. 2015) and have been suggested as the cause of observed UV flares for HD $189733 \mathrm{~b}$ (Pillitteri et al. 2015). Accretion streams or blobs can be viewed in transmission if the optical depth is sufficiently high in the transition of interest.

Besides material transiting ahead of the planet, changes in the stellar activity level can be also produce observable changes in the pre-transit transmission spectrum: if spectra from a period of low activity are compared to spectra from a period of higher activity, the relative difference can produce features similar to what is seen in tranmission spectrum absorption lines. HD 189733 is an active early K-star (Boisse et al. 2009) and, especially for the Balmer line analysis presented here, contributions from the stellar chromosphere may be nonnegligible. These contributions need to be taken into account 
when interpreting transmission spectra of lines produced in stellar active regions.

Evidence for pre-transit material has now been observed in a handful of hot planet systems, namely, WASP-12 b (Fossati et al. 2010), HD 189733 b (Ben-Jaffel \& Ballester 2013; Bourrier et al. 2013; Cauley et al. 2015), and GJ 436 b (Ehrenreich et al. 2015; see Section 1 of Cauley et al. 2015, for a brief overview). Most recently, Ehrenreich et al. (2015) reported a large pre-transit absorption signature, as well as enhanced in-transit absorption, in Ly $\alpha$ around the hot Neptune GJ $436 \mathrm{~b}$. They model the early ingress as an extended cloud of hydrogen which has escaped from the planet. The escaping material is subject to radiation pressure from the star, which in the case of GJ 436 is too weak to overcome the stellar gravity and instead acts as a radiative braking agent on the gas (Bourrier et al. 2015). We note that (Ehrenreich et al. 2015) do not include the interaction of the planetary wind with the stellar wind. In Cauley et al. (2015, hereafter Paper I) we describe a pre-transit absorption measurement in the Balmer lines for the hot Jupiter HD 189733 b (Bouchy et al. 2005). We showed that the absorption strength and time series evolution of the absorption is consistent with the geometry of a thin bow shock at $\sim 13 R_{p}$ ahead of the planet. There are difficulties, however, in maintaining a population of hot neutral hydrogen at large distances from the planet (C. Huang \& D. Christie 2016, private communication). Furthermore, it is unlikely that the densities produced by a compressed stellar wind at typical hot Jupiter orbital distances are high enough in most atomic species to generate the necessary opacity, although escaping planetary material trapped in the magnetosphere may be sufficient (Turner et al. 2016).

Regardless of their precise interpretation, it appears that pretransit absorption signals should be fairly common for hot planets. However, detections of these phenomena seem to be limited to specific atomic transitions; they have not yet been detected using near-UV broadband methods (e.g., Turner et al. 2013; Bento et al. 2014; Zellem et al. 2015) nor, to the best of our knowledge, optical photometry. High-resolution spectroscopic observations are expensive and are limited to the brightest transiting targets. As a result, the detection and characterization of these signals, including their variability, will require a significant observational investment.

In this paper, we present followup observations to the 2013 transit of HD $189733 \mathrm{~b}$ presented in Paper I. Our observations of the 2013 transit missed a large portion of the pre-transit phase due to an observing strategy that was not designed to monitor pre-transit signals. The 2015 transit observations provide complete coverage of the $\sim 4 \mathrm{hr}$ of visible pre-transit phase. The instrumental setup and experimental design are almost identical to the 2013 transit, facilitating a direct comparison between the two measurement sets. In Section 2 we briefly describe the transit observations and data reduction. Section 3 details the construction of the Balmer line, Na I D lines, and $\mathrm{Mg}$ I $5184 \AA$ transmission spectra and the line absorption time series for each line. We present an analysis of the $\mathrm{Ca}$ II $\mathrm{H}$ and $\mathrm{K}$ lines, which serve as a proxy of the stellar activity level, in Section 4. The residual core flux analysis in Section 4 differs from the Mt. Wilson $S_{\mathrm{HK}}$ analysis presented in Paper I and provides a more precise estimate of the relative stellar activity level. Contrary to the $S_{\mathrm{HK}}$ index analysis presented in Paper I, the core flux analysis shows that there is some relationship between the stellar activity level and the measured absorption. We present possible model scenarios to describe the new pre-transit absorption in Section 5. We will present a detailed analysis of the in-transit absorption in a forthcoming paper. In Section 6 we give a general summary of the results and comment on future work that is required to clarify the nature of the pre-transit signature.

\section{OBSERVATIONS AND DATA REDUCTION}

A single transit epoch of HD 189733 b was obtained on the night of 2015 August 4 using HiRES on Keck I (Vogt et al. 1994). The instrument setup and experimental design were identical to the 2013 transit observations described in Paper I. We briefly review them here. The B2 decker was used and the resolving power of the observations is $R \sim 68,000$ at $\mathrm{H} \alpha$. Exposure times were 5 minutes for all observations resulting in an average signal to noise of 450 for the extracted spectra at $\mathrm{H} \alpha, 200$ at $\mathrm{H} \beta$, and 150 at $\mathrm{H} \gamma$. The data were reduced using the HiRES Redux package written by Jason X. Prochaska. ${ }^{4}$ All standard reduction steps were taken and the barycentric velocity of the observatory is removed, along with the radial velocity of the HD 189733 system which we take to be $-2.24 \mathrm{~km} \mathrm{~s}^{-1}$ (Di Gloria et al. 2015), leaving the spectra in the rest frame of the star. Wavelength solutions are performed on Th-Ar exposures taken at the beginning and end of the night. Telluric absorption is removed from the $\mathrm{H} \alpha$ and $\mathrm{Na}$ I spectra using the telluric fitting program Molecfit (Kausch et al. 2014). A telluric standard was observed at the beginning of the night. The telluric model is first applied to the standard and then scaled and shifted to fit each individual observation using a least-squares minimization routine.

\section{TRANSMISSION SPECTRA}

The transmission spectrum is defined here as

$$
S_{T}=\frac{F_{i}}{F_{\text {out }}}-1,
$$

where $F_{i}$ is a single observation and $F_{\text {out }}$ is the master comparison spectrum. The master comparison spectrum is a weighted average of a set of spectra that are, ideally, free of any absorption contamination, i.e., they represent the pure stellar spectrum. In order for each $F_{i}$ to be compared to $F_{\text {out }}$, the spectra need to be normalized and aligned in wavelength space. The required constant wavelength shifts from one observation to the next, which are calculated by cross-correlating a large number of metal lines in the order of interest, are of the order $0.01-0.02 \AA$ or $0.5-1.0 \mathrm{~km} \mathrm{~s}^{-1}$. The corrections applied to the $\mathrm{H} \alpha$ order, relative to the first observed spectrum, are shown in Figure 1. Higher-order functions, e.g., linear or spline fits, are not necessary to align the spectra. After the spectra are aligned and divided by $F_{\text {out }}$ they are renormalized to remove any residual slope in the continuum.

We note that HiRES is a very stable spectrograph and does not produce large instrumental effects on the observed spectrum. This is important for having confidence in the transmission spectrum signal as being due to real changes in the star-planet system and not from instrument and telescope systematics. Furthermore, any systematic shifting of the spectrum on the detector or changes in resolution would

\footnotetext{
4 http://www.ucolick.org/ xavier/HIRedux/
} 


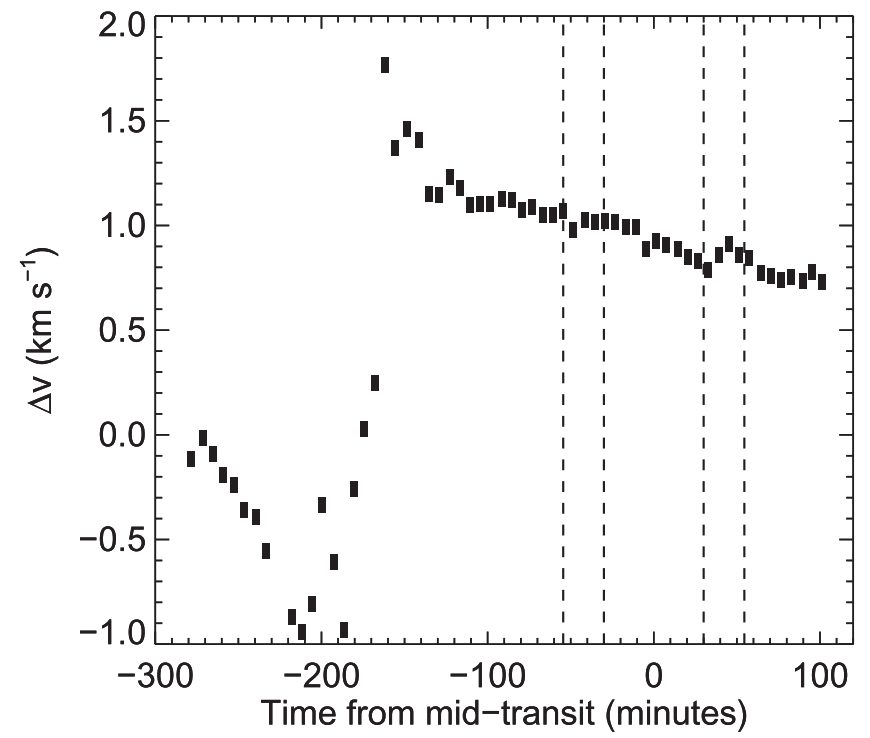

Figure 1. Velocity corrections applied to the $\mathrm{H} \alpha$ order, relative to the first spectrum (not shown) for each observation. The comparison spectrum corrections are shown with red bow ties. The abrupt changes at -200 and -160 minutes are due to small guiding errors that resulted in the telescope being re-centered on the target.

uniformly affect all stellar lines in a single order. This is never observed: all of the transmission line profiles reported here occur only in the cores of the stellar lines of interest and not in the plethora of other stellar lines present in every order. Thus we are confident all of the reported transmission signals are intrinsic to the star-planet system.

In order to create the transmission spectrum, the comparison spectra used to generate the master comparison spectrum must be selected. Since all of the absorption is measured relative to the master comparison spectrum, choosing different spectra will increase or decrease the measured absorption values. Thus the true zero-point is difficult to determine for the small window of time that a single night of observing provides. We have chosen to use the eight spectra from $t-t_{\text {mid }}=$ -278 minutes to $t-t_{\mathrm{mid}}=-233$ as the comparison spectra for the analysis presented below in the rest of Section 3. These spectra are chosen since they are the earliest spectra obtained during the night and are farthest from the planet transit in time. If pre-transit absorption is due to extended structures around the planet, the farther the observations occur from the planet transit the less likely those observations are to contain a signal from optically thick circumplanetary material. Of course, this does not guarantee that the spectra are uncontaminated by such material. Our choice of comparison spectra and the consequences for interpreting the absorption measurements will be discussed in Section 4.2.

\subsection{Average Transmission Spectra}

The average transmission spectra are shown in Figure 2 for $\mathrm{H} \alpha 6562.79 \AA$ (first row), $\mathrm{H} \beta 4861.35 \AA$ (second row), and $\mathrm{H} \gamma$ $4340.47 \AA$ (third row) for the pre-transit (first column), intransit (second column), and out-of-transit comparison observations (third column). The number of spectra used to compute the average spectrum is shown in the bottom right corner of each panel in the first row. The fourth column shows the empirical Monte Carlo (EMC) distributions, which highlight the influence of systematic effects on the absorption measurements, of the absorption for each line. The fifth column shows the EMC for the pre-transit signal. Figure 3 shows the same thing for the $\mathrm{Na}$ I $5895.92 \AA$ and $\mathrm{Mg}$ I $5183.60 \AA$ lines. Due to its location at the very edge of the order, we do not extract and analyze the Na I $5889 \AA$ line.

We perform two different EMC procedures: an In-In and InOut procedure (see Redfield et al. 2008; Jensen et al. 2012; Cauley et al. 2015; Wyttenbach et al. 2015, for similar applications). The Pre-Pre and Pre-Out procedures are identical except for the pre-transit spectra. The In-Out EMC compares randomly selected subsets of the in-transit spectra to the master comparison spectrum. The number of random spectra varies from $N=5$ to $N=18$ and the process is repeated for $N=5000$ iterations. The In-In procedure compares a random subset of in-transit spectra with another random subset of in-transit spectra. The random subsets are chosen without replacement so that spectra are never compared to themselves and the number of random spectra varies from $N=2$ to $N=16$. The In-In distributions should be centered at zero absorption and should be broader than the In-Out distributions due to the effect of comparing two different random subsets instead of one random subset with a fixed comparison spectrum. We choose the standard deviation of the In-Out distribution as the $1 \sigma$ uncertainty associated with the EMC procedure since this is directly related to the variation in the absorption measurement.

As expected, the In-In distributions (green) are centered near zero absorption. The $1 \sigma$ values from the In-Out EMC procedure are shown in black. We also calculate the propagated flux uncertainty associated with the master absorption measurement (vertical red dashed line). The flux uncertainty is the standard deviation of two $50 \mathrm{~km} \mathrm{~s}^{-1}$ wide portions of the transmission spectrum from -250 to $-200 \mathrm{~km} \mathrm{~s}^{-1}$ and +200 to $+250 \mathrm{~km} \mathrm{~s}^{-1}$ weighted by the square root of the normalized observed spectrum in order to account for the larger uncertainties in the line cores. This value is shown in orange. The larger of the EMC and flux uncertainty measurements is used for determining the significance of the absorption. The significance level $n \sigma$ is shown in red next to the master absorption measurement.

The absorption is the negative of the equivalent width of the transmission spectrum integrated from $-200 \mathrm{~km} \mathrm{~s}^{-1}$ to $+200 \mathrm{~km} \mathrm{~s}^{-1}$. More formally,

$$
W_{\lambda}=\sum_{v=-200}^{+200}\left(1-\frac{F_{v}}{F_{v}^{\text {out }}}\right) \Delta \lambda_{v},
$$

where $F_{v}$ is the flux in the spectrum of interest at velocity $v, F_{v}^{\text {out }}$ is the flux in the comparison spectrum at velocity $v$, and $\Delta \lambda_{v}$ is the wavelength difference at velocity $v$. The gray regions in Figure 2 indicate the cores of identified stellar absorption lines other than the line of interest. These regions are ignored when calculating $W_{\lambda}$. The master absorption detections are presented in Table 1.

The in-transit and pre-transit absorption for $\mathrm{H} \alpha$ and $\mathrm{H} \beta$ is detected at $>3 \sigma$, confirming the detections made in Paper I and the $\mathrm{H} \alpha$ detection from Jensen et al. (2012). The level of intransit $\mathrm{H} \alpha$ absorption is almost identical to that from Jensen et al. $\left(2012 ; W_{\mathrm{H} \alpha}=13.7 \times 10^{-3} \AA\right)$ and $2.3 \times$ the value measured in Paper I $\left(W_{\mathrm{H} \alpha}=5.9 \times 10^{-3} \AA\right)$. The in-transit $\mathrm{H} \gamma$ absorption is detected at $4.0 \sigma$; the measurement is marginal $(2.2 \sigma)$ for the pre-transit absorption. 


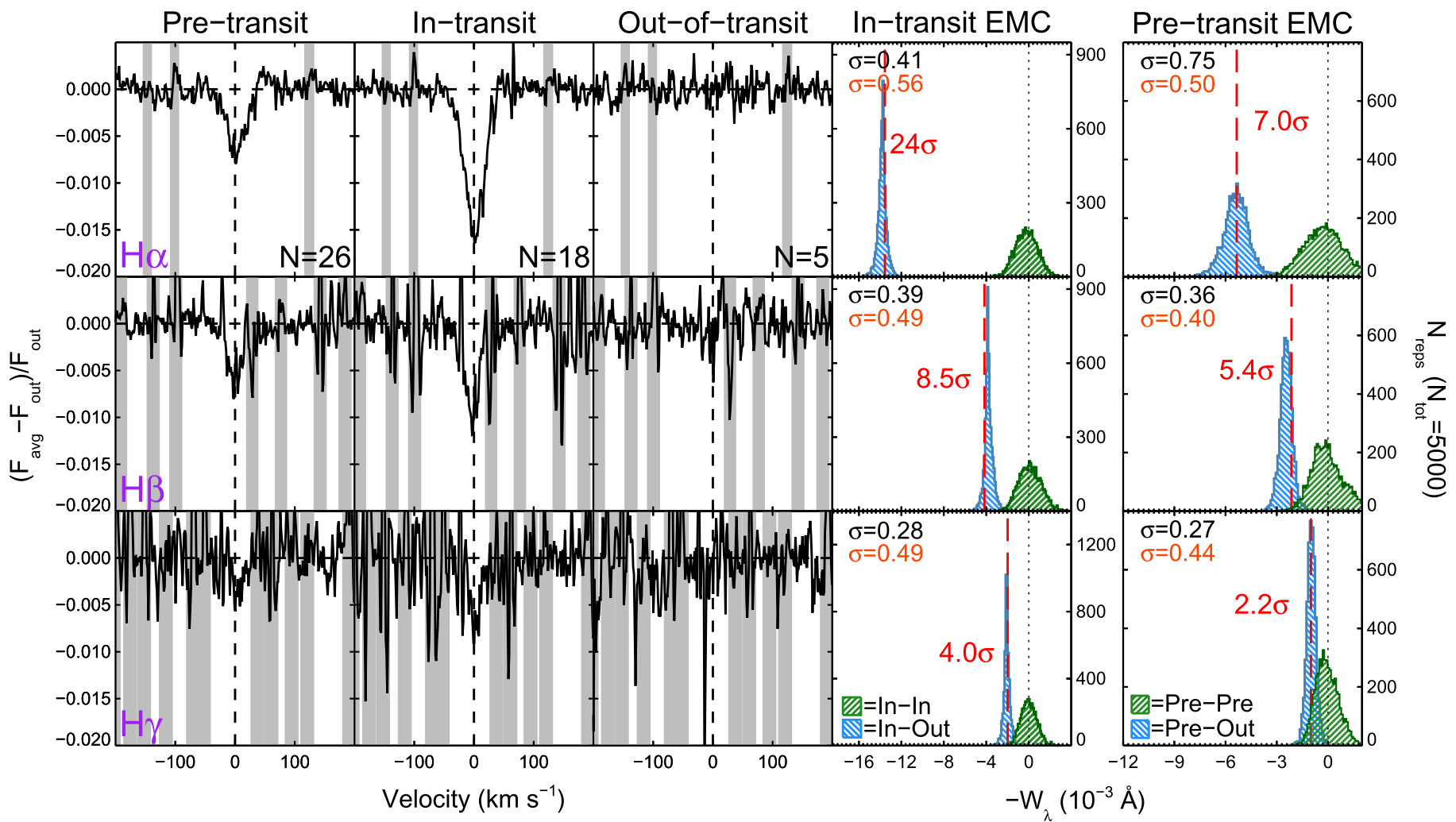

Figure 2. Averaged transmission spectra for the pre-, in-, and post-transit spectra for $\mathrm{H} \alpha$ (top row), $\mathrm{H} \beta$ (middle row), and $\mathrm{H} \gamma$ (bottom row). The cores of identified stellar lines other than the lines of interest are masked in gray. These points are not included in the calculation of the absorbed flux. The number of spectra used to create the average spectrum is listed in the first three panels of the top row. The fourth frame in each row shows the in-transit empirical Monte Carlo (EMC) $W_{\lambda}$ distributions for each line. The master absorption measurement is marked with a vertical dashed red line. The uncertainty in $\mathrm{W} \lambda$ derived from the In-In EMC procedure is marked in black in the upper-left; the propagated flux uncertainty is labeled in orange. The larger of the two is adopted for determining the detection significance. Each in-transit absorption measurement is detected above the $3 \sigma$ level. The $\mathrm{H} \alpha$ and $\mathrm{H} \beta$ pre-transit measurements are detected at $>3 \sigma$.

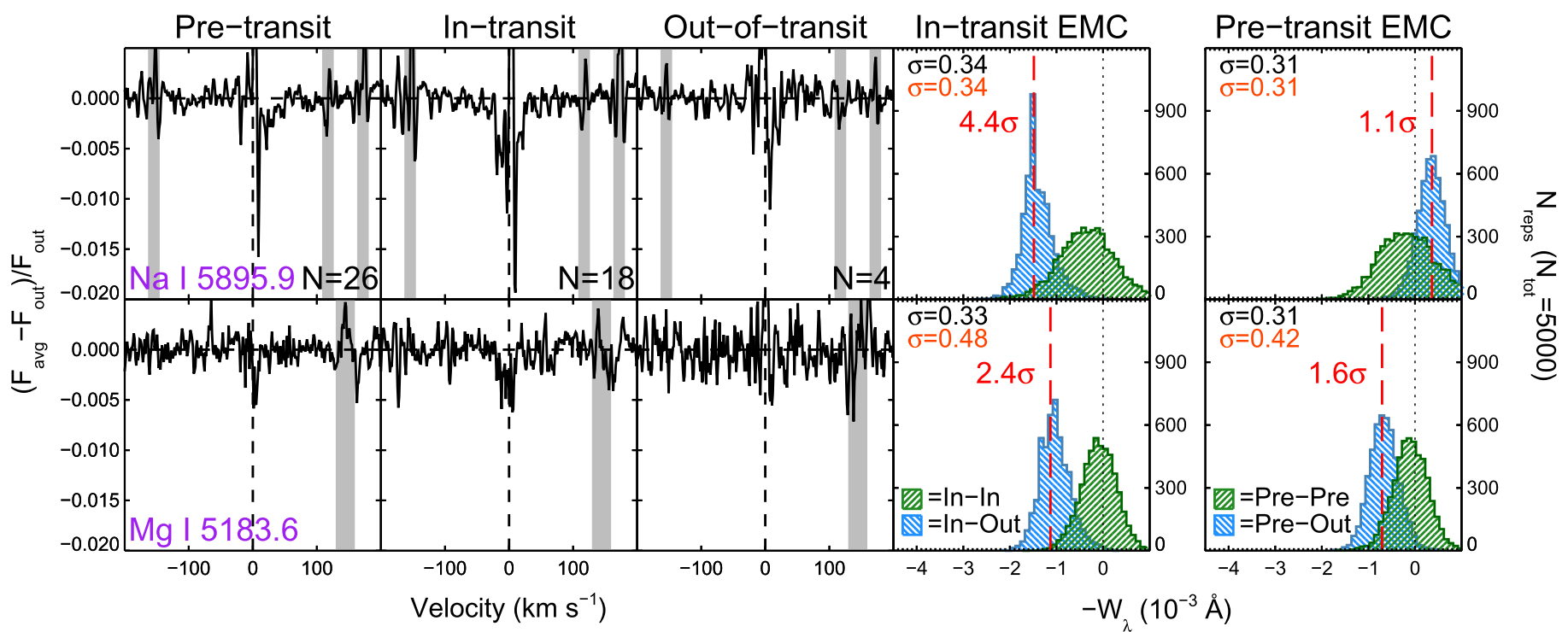

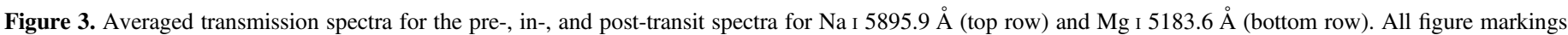

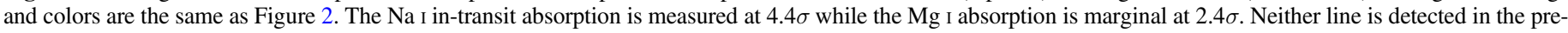
transit transmission spectra.

The in-transit Na I absorption is detected at $4.4 \sigma$ while the $\mathrm{Mg}$ I absorption is marginal at $2.4 \sigma$. There is little evidence for pre-transit absorption in either line. In order to compare our Na I $5896 \AA$ in-transit absorption value to previously reported high-resolution $\mathrm{Na}$ I absorption, we have applied Equation (2) to the Na I $5896 \AA$ transmission spectra presented in Redfield et al. (2008), Jensen et al. (2011), and Wyttenbach et al. (2015). In the case of Wyttenbach et al. (2015), we have used their reported Gaussian fit parameters for the $5896 \AA$ line in order to approximate the absorption. For Jensen et al. (2011) we find 
Table 1

Master Absorption Line Detections

\begin{tabular}{|c|c|c|c|c|}
\hline \multirow[b]{2}{*}{$\begin{array}{l}\text { Spectral Line } \\
\text { (1) }\end{array}$} & \multicolumn{2}{|c|}{ Pre-transit } & \multicolumn{2}{|c|}{ In-transit } \\
\hline & $\begin{array}{c}\left(10^{-3} \AA\right) \\
(2)\end{array}$ & $\begin{array}{c}\sigma \\
\left(10^{-3} \AA\right) \\
(3)\end{array}$ & $\begin{array}{c}\left(10^{-3} \AA\right) \\
(4)\end{array}$ & $\begin{array}{c}\sigma \\
\left(10^{-3} \AA\right) \\
(5)\end{array}$ \\
\hline $\mathrm{H} \alpha$ & 5.25 & 0.75 & 13.44 & 0.56 \\
\hline $\mathrm{H} \beta$ & 2.16 & 0.40 & 4.17 & 0.49 \\
\hline $\mathrm{H} \gamma$ & 0.97 & 0.44 & 1.96 & 0.49 \\
\hline Na I 5896 & 0.34 & 0.31 & 1.50 & 0.34 \\
\hline Mg I 5184 & 0.67 & 0.42 & 1.15 & 0.48 \\
\hline Ca I 6439.1 & $\ldots$ & $\ldots$ & 0.02 & 0.49 \\
\hline Ca I 4878.1 & $\cdots$ & $\ldots$ & 0.40 & 0.33 \\
\hline Ca I 4355.1 & $\cdots$ & $\cdots$ & -0.46 & 0.76 \\
\hline
\end{tabular}

$W_{\mathrm{Na} \text { I }}=2.85 \pm 0.85 \times 10^{-3} \AA$; for Redfield et al. (2008), $W_{\mathrm{Na}}=3.59 \pm 1.61 \times 10^{-3} \AA$; for Wyttenbach et al. (2015), $W_{\mathrm{Na}_{\mathrm{I}}}=2.22 \times 10^{-3} \AA$. Our reported value of $W_{\mathrm{Na}_{\mathrm{I}}}=$ $1.50 \pm 0.34 \times 10^{-3} \AA$ is consistently lower than the previous measurements.

Although the $\mathrm{Mg}_{\text {I }} 5184 \AA$ in-transit absorption is marginal, the measurement is suggestive of a real signal. The $\mathrm{Mg}_{\text {I }} \mathrm{UV}$ transition at $2853 \AA$ has been detected previously for HD 209458 b by Vidal-Madjar et al. (2013), providing evidence that $\mathrm{Mg} \mathrm{I}$ is present at detectable levels in hot Jupiter atmospheres. The HD $209458 \mathrm{~b}$ signal is much stronger (7.5\%) than that reported here. Although the Mg I $5184 \AA$ line arises from an excited $\mathrm{Mg}$ I state and thus the line strength is not favorable compared to the UV resonance line, future investigations should consider examining the Mg I $5184 \AA$ line due to its ease of observability compared to the UV Mg I lines (see Bourrier et al. 2015) and to confirm or reject the measurement presented here.

\subsection{Ca I Control Lines}

Figure 4 shows a set of Ca I control lines that are used to verify the transmission spectrum analysis. Calcium is expected to condense out of hot Jupiter atmospheres and so should not be present in any significant quantity (Lodders 1999). Each of the $\mathrm{Ca}$ I lines is located in the same order as the Balmer line closest to it in wavelength. Figure 4 is identical to Figure 2 except we do not perform a pre-transit EMC for the control lines. Using the flux errors as the $1 \sigma$ uncertainties, none of the control lines are measured at a significant level (see Table 1). The non-detections in the control lines provide reassurance that the measurement algorithms are being properly applied and not resulting in spurious absorption signatures.

\subsection{Absorption Time Series}

Transmission spectra for individual exposures are shown in Appendix A. The values of $W_{\lambda}$ for the individual spectra are shown as a time series in Figures 5-7. Each of the values is calculated using Equation (2). For all measurements, we choose to use the more conservative average flux uncertainties for the individual points rather than the standard deviation of the EMC procedure. In Figure 5, the mean uncertainty for the individual measurements is shown with the solid colored bars in the upper left. Individual $1 \sigma$ uncertainties are shown for $\mathrm{Na}$ I and $\mathrm{Mg}_{\mathrm{I}}$ in Figures 6 and 7.
The pre-transit Balmer line signature in Figure 5 begins abruptly at -217 minutes, which corresponds to a linear distance of $\sim 17 R_{p}$ to the stellar limb, and lasts for $\sim 70$ minutes until it abruptly disappears. It then returns to a similar level after $\sim 40$ minutes and remains relatively constant until immediately before first contact. The in-transit absorption appears to begin immediately before first contact and increases between first and second contact. The abrupt changes in the level of in-transit absorption, e.g., at $-30,5$, and 40 minutes, may be due to the planet transiting the inhomogeneous stellar surface. (The first event begins at $\sim-70$ minutes, or $\sim 15$ minutes before $t_{\mathrm{I}}$. This suggests that the atmosphere is occulting an extended prominence above the stellar disk. This event ends at $t_{\mathrm{II}}$, immediately after the planetary disk fully occults the star. The second event, from $\sim+5$ to +40 minutes, has a duration similar to the first event. The duration of the events correspond to stellar features with a linear extent of $\sim 2$ $R_{p}$. These abrupt changes in the absorbed flux could also be due to changes in the stellar activity level rather than transits of static features. While this cannot be definitively ruled out, we believe this is less likely due to the very similar duration of both events.) The effect of active regions and changes in the stellar activity level on the line absorption will be discussed more fully in Section 4.1.

An interesting feature of the 2015 transit is that the absorption appears to persist immediately post-transit for $\sim 30$ minutes. The subsequent decrease in absorption at $\sim 90$ minutes, and appearance of emission in the line cores of $\mathrm{H} \beta$ and $\mathrm{H} \gamma$, may be due to a change in the stellar activity level, e.g., a mild flaring event. After the sharp increase, the final two points in the time series decrease from the maximum. The linear extent of the feature causing the post-transit absorption is $>3 R_{p}$ if we take the beginning of the small flaring event to be the end of the post-transit absorption. The depth of the measured post-transit absorption indicates a disk coverage fraction larger than the nominal extended atmosphere we use to model the in-transit absorption in Section 5 since the opaque planet is no longer blocking the stellar disk. The post-transit absorption may be the base of the extended evaporative flows previously detected around HD $189733 \mathrm{~b}$ in neutral hydrogen (Lecavelier des Etangs et al. 2010, 2012; Bourrier et al. 2013).

Only one individual $W_{\lambda}$ value at $t-t_{\text {mid }}=-48$ minutes, is detected at $>3 \sigma$ for $\mathrm{Na}$ I. None of the individual absorption values for $\mathrm{Mg}$ I is detected at $>2 \sigma$. There is a notable decrease, however, in the $\mathrm{Na}$ I in-transit points compared to the pre- and post-transit values and the $\mathrm{Mg}$ I values are consistently below zero beginning at $\sim-255$ minutes. The structure of the intransit $\mathrm{Na}$ I absorption shows a similar shape to the center-tolimb variations (CLVs) described by Czesla et al. (2015). CLVs may be important in determining the precise level of atmospheric $\mathrm{Na}$ I absorption and should be taken into account for a detailed analysis of the in-transit absorption. Such an analysis is beyond the scope of this paper. The consistently high post-transit values of $W_{\mathrm{Na} \text { I }}$ in Figure 6 appear to be due to the large core residuals and not to any real emission features.

\subsection{Ho Line Centroid Velocities}

The signal to noise in the individual $\mathrm{H} \alpha$ transmission spectra is high enough to consider the overall velocity shift of the absorption. Measurements of the line velocity can be useful in understanding the mass motions of the absorbing material. In particular, measurements of in-transit line velocities can 


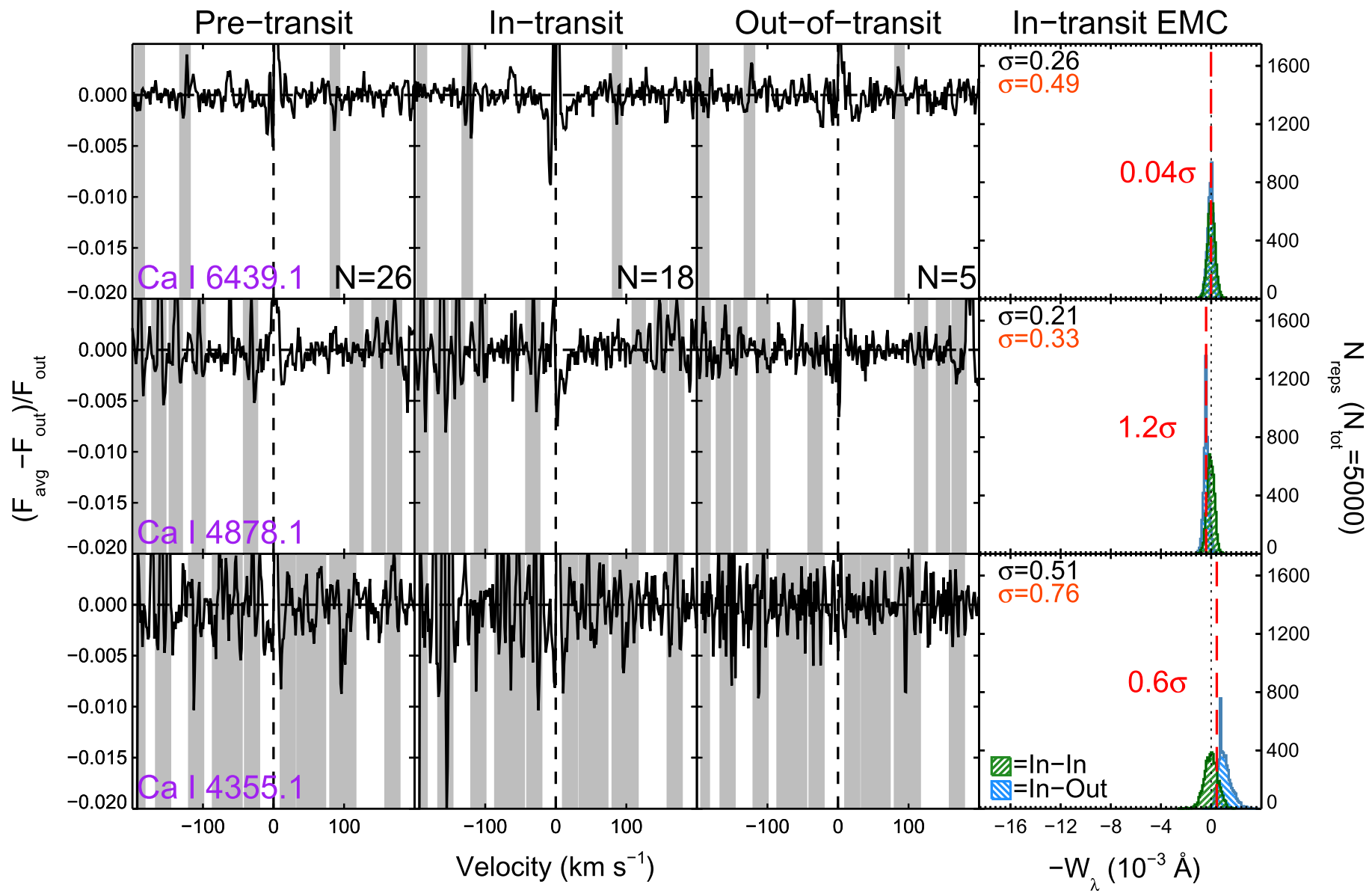

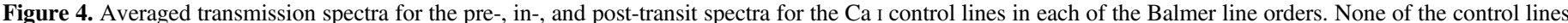

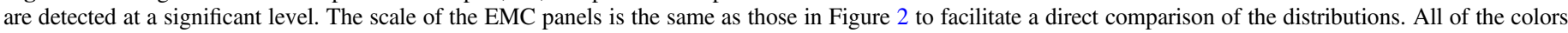
and labels are the same as those in Figure 2.

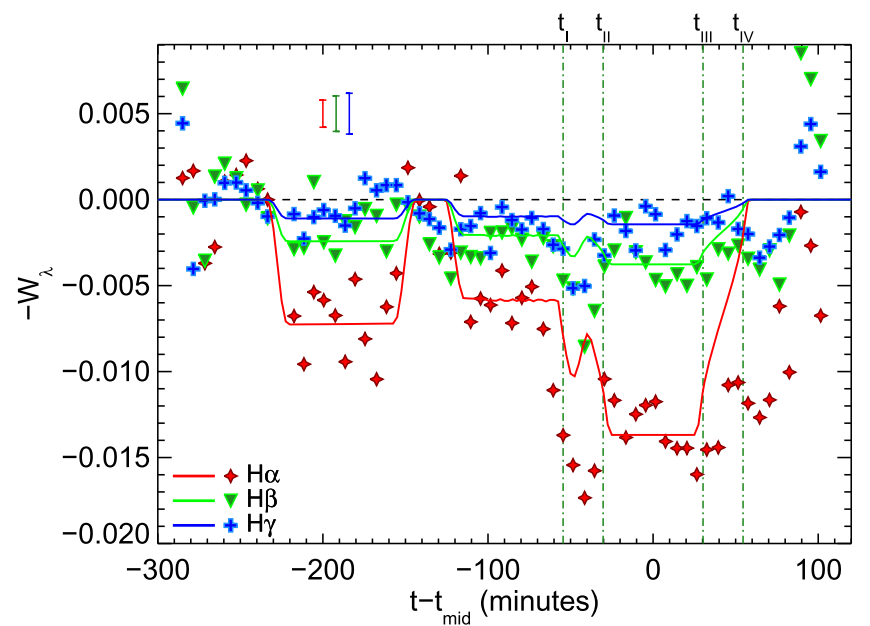

Figure 5. Absorption time series calculated for each of the transmission spectra shown in Figures 16-18. Values of $W_{\lambda}$ are calculated using Equation (2). The average $1 \sigma$ uncertainties in $W_{\lambda}$ for each line are marked with the solid bars in the upper-left. Optical transit contact points are marked with vertical green dashed-dotted lines. The solid lines are model $W_{\lambda}$ values (see Section 5). Note the two distinct pre-transit dips in $W_{\mathrm{H} \alpha}$ between -220 and -155 minutes and then again between -110 and -60 minutes. The in-transit absorption strength is $\sim 2$ times stronger than observed in the 2013 transit and there appears to be sustained absorption for $\sim 30$ minutes post-transit.

provide information about the planetary rotation and atmospheric dynamics (e.g., Showman \& Guillot 2002; Menou \& Rauscher 2010; Rauscher \& Kempton 2014; Snellen

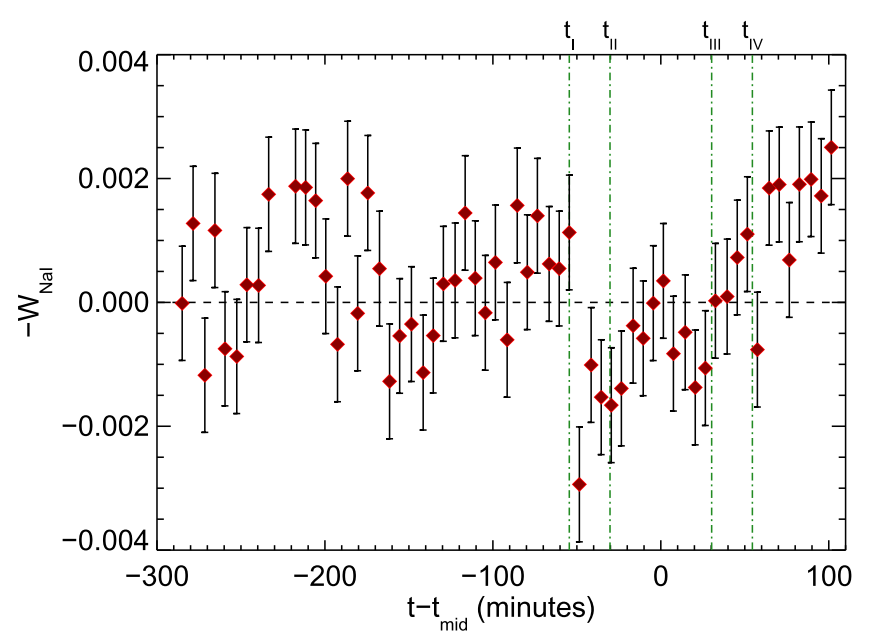

Figure 6. Absorption time series calculated for each of the $\mathrm{Na}$ I transmission spectra shown in Figure 19.

et al. 2014; Brogi et al. 2015; Louden \& Wheatley 2015). Here we will focus on the pre-transit line velocities and a detailed analysis of the in-transit velocities will be presented in a future paper.

The velocity is measured for each $\mathrm{H} \alpha$ transmission spectrum which has a $W_{\lambda}$ value significant at the $2 \sigma$ level. We use a fluxweighted average from -40 to $+40 \mathrm{~km} \mathrm{~s}^{-1}$ to measure the 


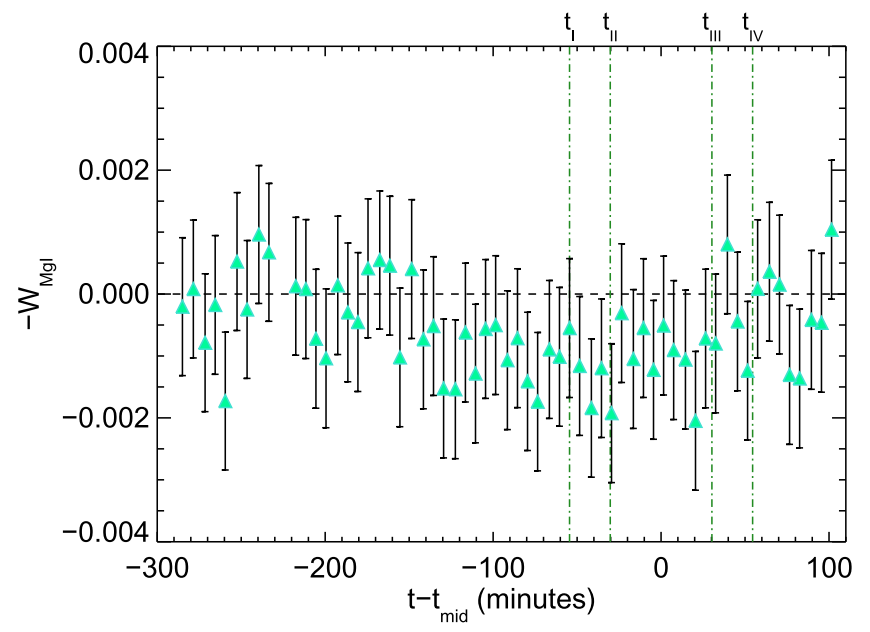

Figure 7. Absorption time series calculated for each of the $\mathrm{Mg}$ I transmission spectra shown in Figure 20.

velocity of the absorption:

$$
v_{\mathrm{H} \alpha}=\frac{\sum_{v=-40}^{+40} v\left(1-f_{v}\right)^{2}}{\sum_{v=-40}^{+40}\left(1-f_{v}\right)^{2}},
$$

where $\left(1-f_{v}\right)^{2}$ is the square of the transmission spectrum flux at velocity $v$. This ensures that deeper portions of the line are heavily weighted. We also add back in the velocity corrections from Figure 1. We note that these corrections are small $(\sim-1.0$ to $1.0 \mathrm{~km} \mathrm{~s}^{-1}$ ) and do not significantly affect any of the conclusions based on the line velocities discussed here. The uncertainty in each velocity is the standard deviation of the distribution derived from calculating the line velocity for each of the $N=255$ combinations $(N=511$ for the 2013 data set) used to produce the individual spectra in Figure 16. This is essentially the EMC process introduced in Section 3.3. We add an extra $1.0 \mathrm{~km} \mathrm{~s}^{-1}$ to each uncertainty to approximate the error in the original velocity correction.

The $\mathrm{H} \alpha$ velocities for both the 2013 and 2015 data sets are shown in Figure 8. Most of the 2015 pre-transit velocities are redshifted, indicating material moving away from the observer. However, immediately before the transit the velocities are grouped relatively close to zero with a sharp transition down from $\sim+4 \mathrm{~km} \mathrm{~s}^{-1}$ between $t-t_{\text {mid }}=-85$ and -79 minutes. There is no obvious explanation for this abrupt change in the line velocity. Also note the consistent blueshift of the 2013 intransit points compared to the symmetric in-transit velocities of the 2015 data. We will revisit the pre-transit velocities during Section 5 in the context of the clumpy accretion stream model.

\section{THE RESIDUAL CA II H CORE FLUX}

The $\mathrm{Ca}$ II $\mathrm{H}$ and $\mathrm{K}$ lines are frequently used as stellar activity indicators (e.g., Duncan et al. 1991; Wright et al. 2004; Isaacson \& Fischer 2010; Gomes da Silva et al. 2014) and are standard diagnostics of solar flare energetics and dynamics (e.g., Johns-Krull et al. 1997; Garcá-Alvarez et al. 2005; Lalitha et al. 2013). $\mathrm{H} \alpha$, which is in emission in stellar active regions, is also used as an activity measure (Meunier \& Delfosse 2009; Gomes da Silva et al. 2014; Kuridze et al. 2015) and correlates directly with $\mathrm{Ca}$ II $\mathrm{H}$ and $\mathrm{K}$ across solar cycles (Livingston et al. 2007). Much variation exists, however, in the

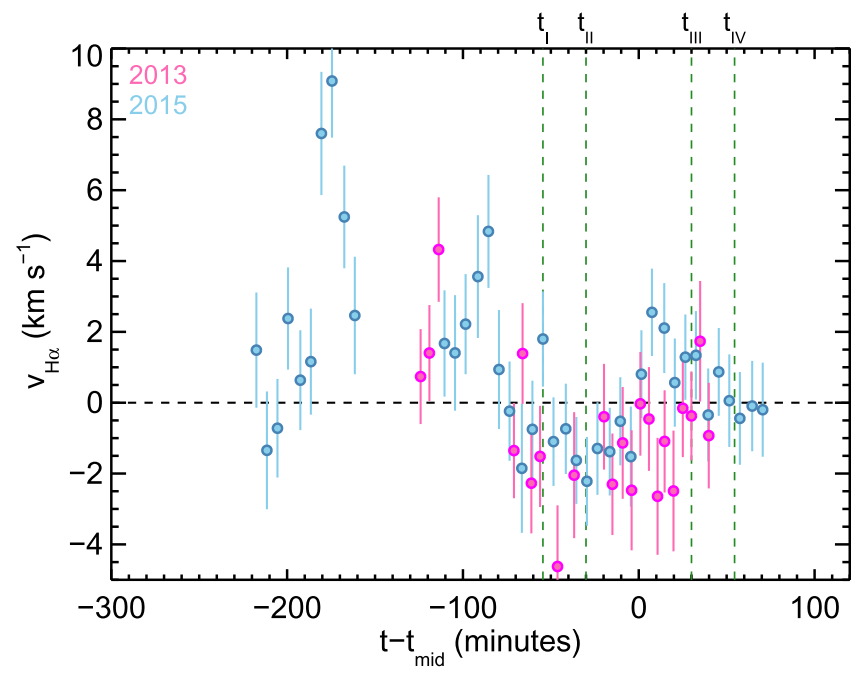

Figure 8. Line centroid velocities calculated for $\mathrm{H} \alpha$ transmission spectra that display $2 \sigma$ absorption in Figure 16. Values calculated for the 2013 data from Paper I are also shown. The green vertical dashed lines show the transit contact points. The full range of $t-t_{\text {mid }}$ is shown for easy comparison with Figure 5. The pre-transit lines show mostly positive velocities, indicating material moving toward the star. An analysis of the in-transit velocities will be presented in a future paper.

$\mathrm{H} \alpha-\mathrm{Ca}$ II $\mathrm{H}$ and $\mathrm{K}$ correlation for other stars (e.g., Cincunegui et al. 2007). Surface $\mathrm{H} \alpha$ activity can therefore mimic an absorption signature: if the comparison spectra capture the star in a more active state compared to the spectra of interest (e.g., the pre- or in-transit spectra), the filling in of the line cores in the active spectra will result in "absorption" in the transmission spectrum of a non-active spectrum. Thus it is critical to understand the stellar activity level as a function of time in order to attempt to separate any activity contribution to the absorption signature.

While measurements of $\mathrm{Ca}$ II and $\mathrm{H} \alpha$ core flux have been used to study the long term (days to years) influence of hot planets on their host stars (e.g., Shkolnik et al. 2005, 2008; Fares et al. 2010; Scandariato et al. 2013), there has been little effort to characterize the very short term variations (minutes to hours) in the line cores of stars other than the Sun. Ideally, short-cadence observations of the star would be carried out at multiple phases of a single planetary orbit in order to establish a baseline of the short-term stellar activity level and characterize typical changes in the line core flux. This baseline could be used to separate the purely stellar component from any variations caused by circumstellar material during the phase of interest.

$\mathrm{H} \alpha$ and $\mathrm{Ca}$ II $\mathrm{H}$ and $\mathrm{K}$ emission is spatially coincident in active regions on the Sun (e.g., see Meunier \& Delfosse 2009; Kuridze et al. 2015), although the two line fluxes do not necessarily correlate across the entire solar disk. Filaments, which are cooler than plages and can absorb active region $\mathrm{H} \alpha$ emission, can affect the level of observed $\mathrm{H} \alpha$ flux and weaken the correlation between $\mathrm{H} \alpha$ and $\mathrm{Ca}$ II $\mathrm{H}$ and $\mathrm{K}$ (Meunier \& Delfosse 2009). Short cadence observations of flaring regions on the Sun show a direct correlation between $\mathrm{Ca}$ II and $\mathrm{H} \alpha$ excess emission equivalent widths (e.g., Johns-Krull et al. 1997; Garcá-Alvarez et al. 2005). A similar correspondence is seen in dMe flares. In the case of dMe objects, the peak Ca II $\mathrm{K}$ flux occurs $\sim 30$ minutes after the peak in the Balmer line fluxes and variations of $\sim 5 \%$ are common on 10 - 


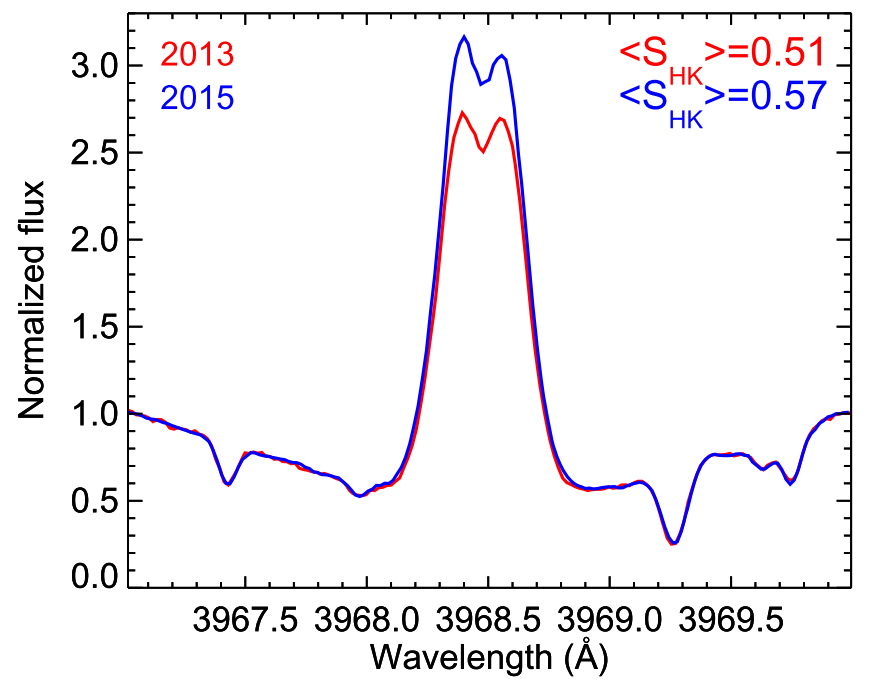

Figure 9. Comparison of the normalized $\mathrm{Ca}$ II $\mathrm{H}$ line cores of the comparison spectrum for the 2013 transit (red) and 2015 transit (blue). The 2015 line core has $\sim 12 \%$ more flux than the 2013 line core, indicating that the star is in a more active state during the 2015 transit.

minute timescales (Kowalski et al. 2013). Although HD 189733 is not as active as a typical dMe star, it is an active K-dwarf and so it is likely that it would exhibit low-level chromospheric variability on short timescales. To the best of our knowledge, no continuous short-cadence observations of HD 189733's $\mathrm{H} \alpha$ and the $\mathrm{Ca}$ II $\mathrm{H}$ and $\mathrm{K}$ lines, similar to the observations presented here and in Paper I, have been published. Thus it is difficult, outside of the transits presented in this work, to quantify the stellar contribution to variations in the line fluxes on short timescales.

In Paper I, we used the time series structure of the $\mathrm{Ca}$ II $\mathrm{Mt}$. Wilson $S_{\mathrm{HK}}$ index to argue that the pre-transit signal was not caused by varying stellar activity and was instead due to material occulting the star. This argument was based on the lack of correlation between the $S_{\mathrm{HK}}$ index and $W_{\mathrm{H} \alpha}$. Furthermore, the mean $S_{\mathrm{HK}}$ value of the comparison spectra was almost identical to that of the pre-transit spectra, suggesting a similar activity level pre- and post-transit. However, the $S_{\mathrm{HK}}$ index compares the core flux with a wide continuum window and, as a result, is subject to variations in the continuum flux. Small variations in the continuum window flux, which are not necessarily related to the same physical processes causing flux changes in the line cores, can thus mimic changes in the stellar activity level.

In order to mitigate any contribution to the activity index by the continuum windows, we have reanalyzed the $2013 \mathrm{Ca}$ II lines using the residual core flux method of Shkolnik et al. (2005). We have also applied this analysis to the $2015 \mathrm{Ca}$ II lines. To be clear, the $S_{\mathrm{HK}}$ index is well suited for measuring average activity levels across different epochs and, indeed, we present the average $S_{\mathrm{HK}}$ values for each night in Figure 9. However, a more precise measurement of variations in the stellar activity level, is better achieved by measuring only the core flux.

The residual profiles are constructed by normalizing the line core to $0.1 \AA$ wide spectral regions centered at 3967.05 and $3969.95 \AA$ and subtracting the mean profile of the same comparison spectra used to generate the average Balmer line transmission spectra. Individual values of $W_{\mathrm{CaH}}$ are calculated

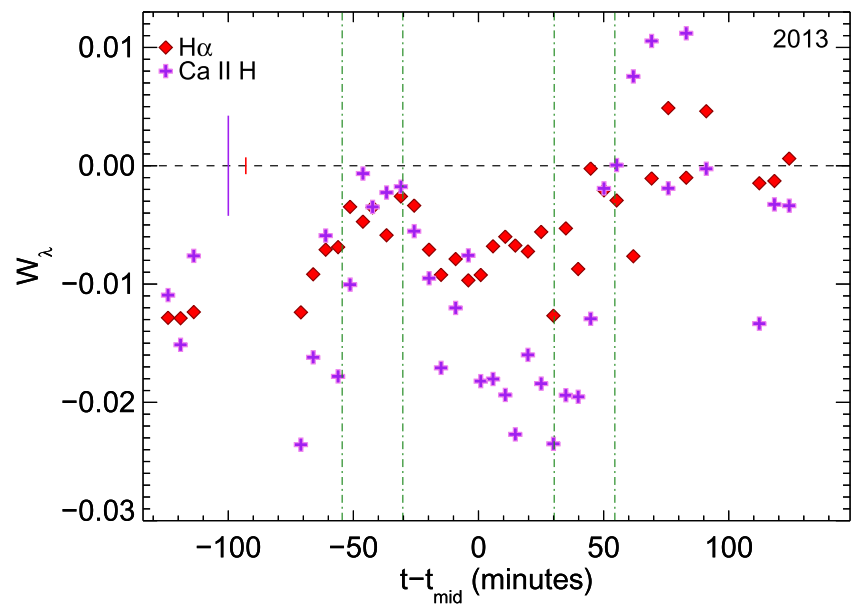

Figure 10. Ca II $\mathrm{H}$ residual core flux time series for the 2013 transit. The $W_{\mathrm{H} \alpha}$ values are over plotted for comparison. Typical $1 \sigma$ uncertainties are shown with solid colored lines in the upper left. The core flux alone shows different behavior than the $S_{\mathrm{HK}}$ index used in Paper I. Notably, the core flux appears to trace the $\mathrm{H} \alpha$ absorption, suggesting that some of the signal in $\mathrm{H} \alpha$ attributed to absorption may be caused by varying stellar activity. In addition, the residual core flux of the comparison spectra (the nine post-transit spectra) is higher than the pre-transit spectra. However, the correlation is weak (see Figures 12 and 13) so it is difficult to quantify how much of the signal is purely due to stellar activity.

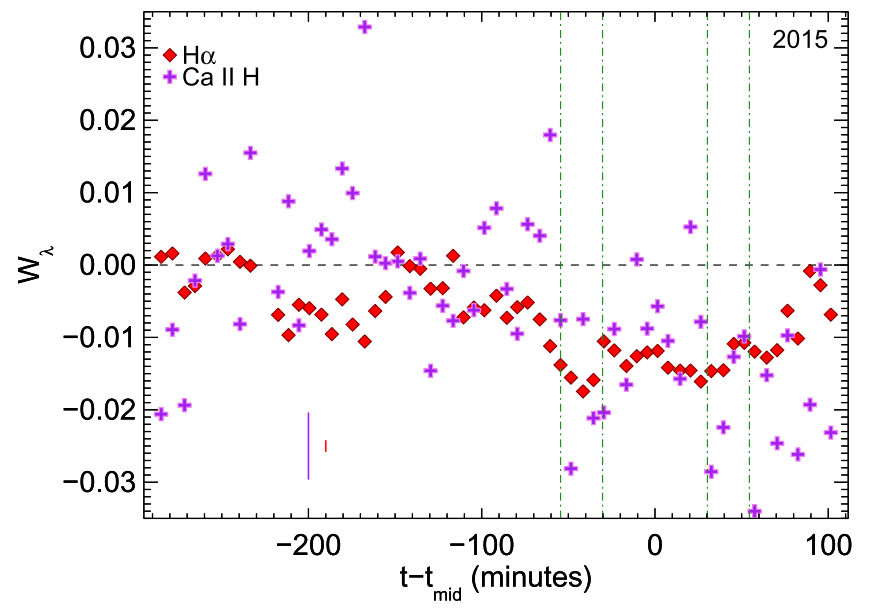

Figure 11. Same as Figure 10, except for the 2015 transit. Note the larger plot range for $W_{\lambda}$. The scatter in $W_{\mathrm{CaH}}$ is larger than for the 2013 transit and there is little correlation between $W_{\mathrm{CaH}}$ and $W_{\mathrm{H} \alpha}$ (see Figure 12), although the mean intransit value of $W_{\mathrm{CaH}}$ is lower than the mean pre-transit value. Note the low values of $W_{\mathrm{CaH}}$ for the first three comparison spectra near $\sim-280$ minutes.

by summing over the residual spectrum from 3967 to $3970 \AA$. The mean $\mathrm{Ca}$ II $\mathrm{H}$ profiles for the comparison spectra from each night are shown in Figure 9. The $\mathrm{Ca}$ II $\mathrm{H}$ residual profiles are shown for the 2013 transit in Figure 21 and for the 2015 transit in Figures 22 and 23. We exclude the $\mathrm{Ca}$ II $\mathrm{K}$ profiles due to lower signal to noise. ${ }^{5} \mathrm{We}$ note that the residual profiles are not identical to the transmission spectra, since they are not normalized by the comparison spectrum, and so values of $W_{\mathrm{H} \alpha}$ and $W_{\mathrm{CaH}}$ cannot be directly compared.

Figures 10 and 11 show the $W_{\mathrm{CaH}}$ timeseries for the 2013 and 2015 transits, respectively. The values of $W_{\mathrm{H} \alpha}$ from each transit are shown for comparison. Representative $1 \sigma$

\footnotetext{
5 The $\mathrm{Ca}$ II $\mathrm{H}$ and $\mathrm{K}$ lines are located near the edges of separate orders in our spectrograph setup.
} 

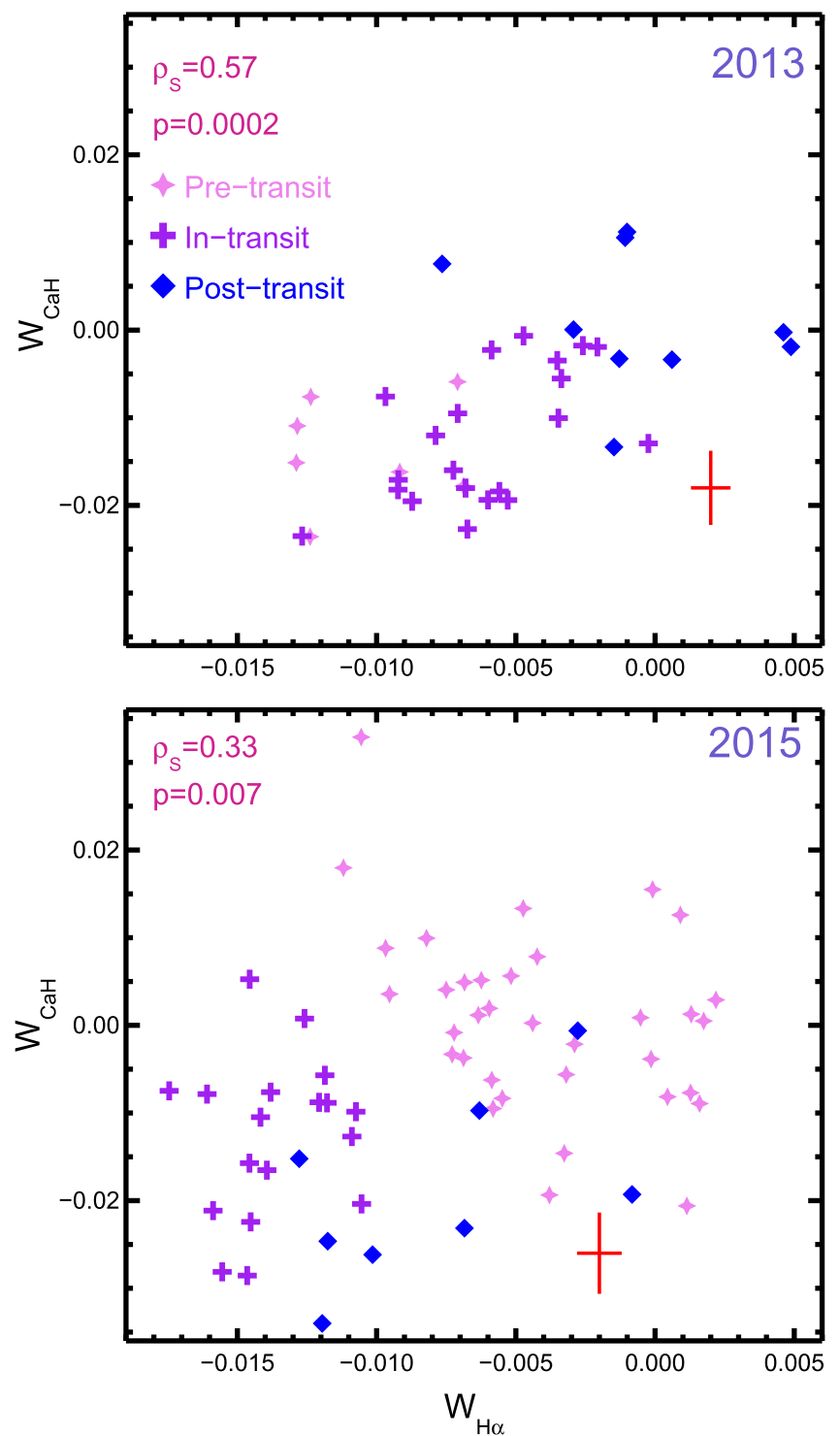

Figure 12. $W_{\lambda}$ for $\mathrm{Ca}$ II $\mathrm{H}$ vs. $\mathrm{H} \alpha$ for the 2013 and 2015 transits. Note the larger plot range for $W_{\mathrm{CaH}}$ in the bottom panel. There is a modest correlation in the 2013 data but it is driven almost entirely by the in-transit points $\left(\rho_{s}=0.52\right.$, $p=0.016$; green crosses). There is a weak but significant correlation in the 2015 data. Typical $1 \sigma$ uncertainties in $W_{\lambda}$ are shown with the red solid lines.

uncertainties are shown with the solid colored bars. The residual core flux in Figure 10 shows different structure compared to the $S_{\mathrm{HK}}$ index from Paper I (see Figure 4 from Paper I). Notably, the pre-transit $W_{\mathrm{CaH}}$ values appear to trace the pre-transit $W_{\mathrm{H} \alpha}$ values and the mean pre-transit $W_{\mathrm{CaH}}$ value is lower than the mean post-transit value, suggesting similar levels of stellar activity both pre- and post-transit. This contradicts the behavior of the $S_{\mathrm{HK}}$ index from Paper I which showed very similar pre- and post-transit values. As described above, this must be due to small changes in the continuum window flux used in the index calculation since the core flux remains the same in both measurements. The $2013 W_{\mathrm{CaH}}$ values are plotted against the $W_{\mathrm{H} \alpha}$ values in the top panel of Figure 12. The Spearman's $\rho$ value, $\rho_{S}$, and the corresponding $p$-value are given in the upper left of the panel. There is a moderate correlation between $W_{\mathrm{CaH}}$ and $W_{\mathrm{H} \alpha}$, although we note that this is mainly driven by the in-transit values (green crosses) which have $\rho_{S}=0.51(p=0.016)$. The $\mathrm{Ca}$ II $\mathrm{H}$ residual values for the

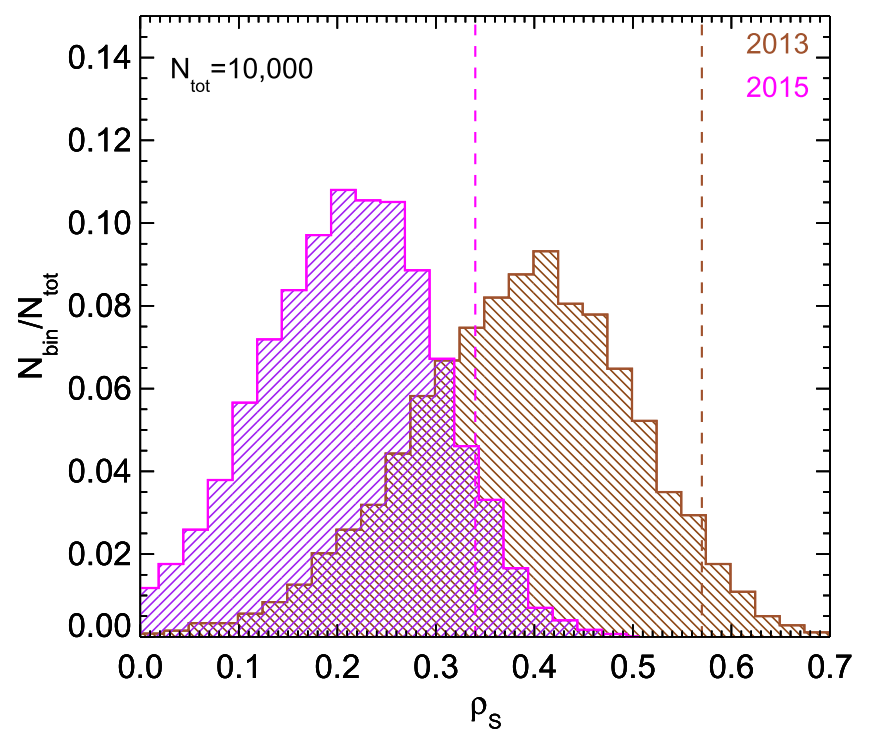

Figure 13. Distributions of $\rho_{s}$ for the $W_{\mathrm{H} \alpha}$ vs. $W_{\mathrm{CaH}}$ correlations shown in Figure 12. The distributions are generated by running 10,000 simulations and letting the values of $W_{\mathrm{CaH}}$ and $W_{\mathrm{H} \alpha}$ vary according to their $1 \sigma$ uncertainties, which are assumed to be normal errors. The measured values of $\rho_{S}$ for both dates (vertical dashed lines) are much higher than the average values of the distributions, suggesting that the true correlations are weaker than the measured correlation.

2015 transit are shown in Figure 11. The $2015 W_{\mathrm{CaH}}$ and $W_{\mathrm{H} \alpha}$ do not show any obvious correspondence, although the intransit $W_{\mathrm{CaH}}$ values are lower on average than the pre-transit values. There is a weak but significant correlation present between the 2015 values of $W_{\mathrm{CaH}}$ and $W_{\mathrm{H} \alpha}$.

In order to test the significance of the correlations seen in both data sets, we have run a simple Monte Carlo procedure that randomly draws values of $W_{\mathrm{CaH}}$ and $W_{\mathrm{H} \alpha}$ from a normal distribution defined by the $1 \sigma$ flux errors for each point. Representative values of the uncertainties are shown with the solid red lines in Figure 12. A simulation of 10,000 random draws is shown in Figure 13 where the 2015 distribution of $\rho_{S}$ is shown in magenta and the 2013 distribution is shown in brown. The nominal $\rho_{S}$ values calculated for the measurements in Figures 10 and 11 are marked with vertical dashed lines. It is clear that the nominal values lie on the high end of the distribution for both dates, suggesting that the true correlation between $W_{\mathrm{CaH}}$ and $W_{\mathrm{H} \alpha}$ is weaker than the nominal values indicate.

While we have focused on stellar activity being the main driver of the $\mathrm{Ca}$ II core flux, it is also possible that the changes in the core flux are in part due to absorption in the pre-transit material. This would weaken any interpretation of the $W_{\mathrm{Ca} \mathrm{II}}-W_{\mathrm{H} \alpha}$ correlation as testing the relationship between stellar activity in the two lines. On the other hand, any absorption by pre-transit material in one line but not the other has a similar effect. Thus interpretation of the $\mathrm{Ca}$ II residual spectrum will also benefit from the activity baseline observations suggested for $\mathrm{H} \alpha$ earlier in this section.

\subsection{The Impact of Stellar Activity}

The analysis presented in Section 4 suggests that the impact of stellar activity on the Balmer line transmission signal is nonnegligible and needs to be taken into account. The correlation between $W_{\mathrm{CaH}}$ and $W_{\mathrm{H} \alpha}$, although modest, weakens the interpretation of the pre-transit signal presented in Paper I as 
being caused by material compressed in a bow shock as opposed to variations in the stellar activity level. Due to the level of scatter in the $W_{\mathrm{CaH}}$ values, and also the fact that $\mathrm{Ca}$ II and $\mathrm{H} \alpha$ do not track exactly the same physical conditions or locations in the stellar chromosphere, we cannot empirically separate the contribution of normal stellar activity from the observed $W_{\lambda}$ time series.

In lieu of an empirical method for separating the stellar activity level from any true absorption, we can explore the parameter space of active region surface coverage and emission line intensity required to reproduce the observed changes in $W_{\lambda}$. We take an approach similar to that of Berta et al. (2011) and examine differences between weighted combinations of active region spectra, i.e., emission lines, and quiet star spectra, in this case a flat continuum. The emission lines are Gaussians with FWHM $\sigma$ and height $A_{0}$. We focus on $\mathrm{H} \alpha$ and take $\sigma=0.79 \AA$, the mean FWHM of the pre-transit absorption lines. The height $A_{0}$ is varied from 0.1 to 1.0 . Low values of the emission line heights are chosen to mimic the low levels of variation that we see here. Moderate solar and stellar flares can cause increases in the emission line flux by factors of $\sim 2$ relative to the local continuum (Johns-Krull et al. 1997; Kowalski et al. 2013) and we do not see evidence (e.g., sharp increases in line strength followed by gradual decay) for large flaring events in our spectra. In addition, large emission line strengths are not necessary to reproduce the observed values of $W_{\mathrm{H} \alpha}$, as shown below.

Active region surface area coverage of the observable hemisphere is varied from $0.1 \%$ up to $10 \%$ (e.g., Andretta \& Giampapa 1995; Meunier \& Delfosse 2009). Spot coverage for HD 189733 has been estimated at $\sim 1 \%$ (Winn et al. 2007). For the Sun, Shapiro et al. (2015) showed that the disk spot coverage fraction $\left(A_{S}\right)$, during peaks in the solar activity cycle, is $\sim 0.2 \%$ while the disk active region coverage fraction $\left(A_{F}\right)$ is $\sim 3 \%$. They also showed that the ratio of $A_{S} / A_{F}$ increases as the level of solar activity increases. This suggests that for HD 189733 the ratio $A_{S} / A_{F}$ is greater than that for the Sun, although the precise relationship is uncertain for very active stars. For the Sun during an activity maximum, $A_{S} / A_{F}=0.07$. Thus we can assume with some confidence that the maximum active region coverage fraction of HD $189733 \mathrm{~b}$ is less than $15 \%$. Furthermore, Shapiro et al. (2015) showed that brightness changes in more active stars are dominated by spots rather than faculae (i.e., bright active regions), although this does not rule out active region variability as the cause of small changes in spectral line flux.

The transmission spectrum is calculated as

$$
S_{T}=\frac{S_{*}\left(1-f_{\mathrm{act}}\right)+f_{\mathrm{act}} S_{\mathrm{act}}}{S_{*}},
$$

where $f_{\text {act }}$ is the fraction of the observable hemisphere covered by active regions with the corresponding spectrum $S_{\text {act }}$. The quiet star spectrum is $S_{*}$, which can be considered to be the comparison spectrum. $W_{\mathrm{H} \alpha}$ is then calculated according to Equation (2).

Figure 14 shows the dependence of $W_{\mathrm{H} \alpha}$ on the active region surface coverage and the strength of the active region emission lines. The average observed value of $W_{\mathrm{H} \alpha}$ for both dates is shown with a horizontal dashed line. The strength of the active region emission line, $A_{0}$, is degenerate with $f_{\text {act }}$. This results in many different combinations of $A_{0}$ and $f_{\text {act }}$ being able to reproduce the observed changes in $W_{\mathrm{H} \alpha}$. Regardless of the

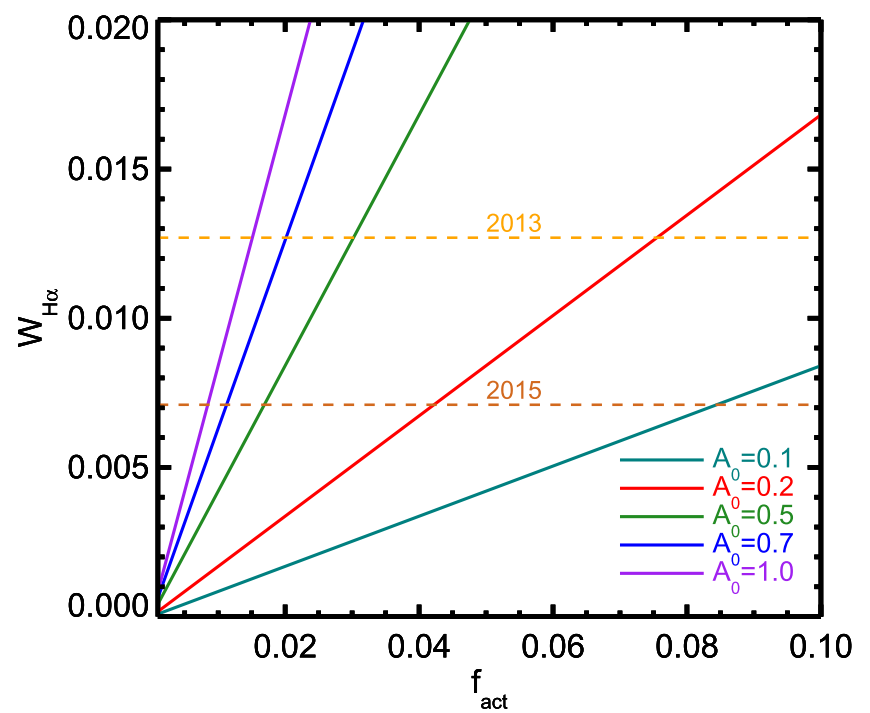

Figure 14. $W_{\lambda}$ as a function of active region surface area fraction $\left(f_{\text {act }}\right)$ for various normalized emission line strengths $\left(A_{0}\right) . W_{\lambda}$ is computed according to Equation (2) for the transmission spectra defined in Equation (4). The horizontal dashed brown line is the mean value of $W_{\mathrm{H} \alpha}$ for the pre-transit absorption values in Figure 5; the dashed orange line is the mean value of the first three pre-transit points from Paper I. While the strength of the emission line is degenerate with $\mathrm{f}_{a c t}$, it is clear that for a range of reasonable parameters the measured change in the pre-transit values of $W_{\lambda}$ for both the 2013 and 2015 transits can be accounted for by low levels of stellar activity.

specific values of $A_{0}$ and $f_{\text {act }}$, the measured levels of pre- and in-transit absorption in both the 2013 and 2015 data can be approximated by relatively small changes in the stellar activity level between the comparison spectra and the spectrum of interest. The smaller pre-transit values for the 2015 transit can be reproduced by almost any combination of $f_{\text {act }}$ and $A_{0}$. The large values from the 2013 transit can be reproduced by most of the parameter combinations but weak line strengths require large surface coverage fractions. Although HD 189733 is an active star, it is unclear whether $8 \%-10 \%$ of the visible hemisphere can be covered in bright active regions. Highcadence monitoring of HD 189733 while HD $189733 \mathrm{~b}$ is not transiting will provide a better statistical understanding of activity variations on $\sim 5$ minutes timescales and help determine the true nature of the pre-transit signal.

For in-transit measurements, contrast between the comparison spectra, which are integrated across the entire stellar disk, and the in-transit spectrum of interest, which is integrated across the stellar disk not covered by the opaque planet, can result in line strength differences as the spectrum is weighted toward active or spotted regions of the star (Berta et al. 2011). The contrast effect can be demonstrated by a planet with no atmosphere transiting a chord that is free of spots or activeregions. In this case, the in-transit spectrum will be weighted toward the spotted or active stellar surface by the ratio $\left(R_{p} / R_{*}\right)^{2}$. For the HD 189733 system, $\left(R_{p} / R_{*}\right)^{2}=0.024$. If the out-of-transit spectrum is composed of $1.00 \%$ spotted/active region spectrum, the weighting of the in-transit spectrum, ignoring limb darkening, will change to $1.02 \%$ spotted/active and $98.98 \%$ quiescent. Thus any difference between the spotted/active region spectrum and the quiescent stellar spectrum will affect the transmission spectrum at the level of $0.02 \%$. This is much smaller than the observed in-transit line depth. We note that this effect changes weakly with increased spot/active region coverage and is $<1 \%$ even for coverage 
fractions of $30 \%$. In addition, the in-transit signal begins before the optical transit and continues after the opaque planetary disk leaves the stellar disk. This is further evidence that the absorption is caused by gas in the planet's atmosphere and not the contrast effect since the contrast effect requires some of the stellar surface to be blocked by the planet.

\subsection{The Choice of Comparison Spectra}

The analysis in Section 4.1 shows that differing levels of stellar activity at different times during the period of observation can affect the relative level of measured absorption in the line core. This is directly related to the choice of comparison spectra. If instead of choosing spectra numbers 2-9 for the 2015 transit we select the observations between $t-t_{\text {mid }}=-230$ and -160 minutes as the comparison spectra, all of the measured $W_{\lambda}$ (Figure 5) values would shift up by the relative average difference between the points in those time ranges. Thus instead of pre-transit absorption we would observe an initial period of enhanced stellar activity, followed by a relative period of quiescence, then another brief activity increase between $t-t_{\text {mid }}=-150$ and -116 minutes, and finally relative quiescence immediately before the transit. If instead we chose the post-transit spectra, as we chose to do in Paper I, the in-transit absorption would be very weak and all of the pre-transit $W_{\lambda}$ measurements would be positive, suggesting sustained levels of higher stellar activity compared to in- and post-transit times. Thus clearly the choice of comparison spectra can influence the interpretation of the $W_{\lambda}$ values.

Our main justification for choosing the earliest observations in the 2015 data as the comparison spectra is that they are located furthest from the transit and thus have a lower likelihood of being contaminated by high-density pre-transit material capable of producing an absorption signature. It would not be unreasonable, however, to select a different set of pretransit spectra. We believe the low post-transit $W_{\lambda}$ values, and their significant variability, rule these spectra out as good outof-transit baseline values. The up-and-down nature of the 2015 pre-transit $W_{\lambda}$ values do not offer much evidence for or against absorbing material versus changes in the stellar activity level as the cause of the signal. However, if the large pretransit variations of $\sim 0.005-0.007 \AA$ at $-230,-155$, and -110 minutes were caused by changes in the stellar activity level, this would indicate such variations are common and we might expect to see similar changes on similar timescales during and after the transit. This is not the case: most of the intransit variations are of smaller magnitude $(\sim 0.003-0.005 \AA)$ and shorter timescales, which may be due to transits of active regions. Furthermore, the duration of the dip in $W_{\mathrm{H} \alpha}$ starting at -220 minutes is $\sim 80$ minutes which is very close to the transit duration of a "narrow" feature. It would be surprising if the star's activity level happened to abruptly change on similar timescales as the transit duration.

Finally, although the individual $W_{\mathrm{H} \beta}$ values are marginal, if the pre-transit spectra between -230 and -155 minutes or between -110 and -60 minutes are chosen as the comparison spectra, the in-transit $\mathrm{H} \beta$ absorption becomes much weaker or disappears entirely. Our results demonstrate the need for a longer baseline of short cadence observations in order to establish the true out-of-transit stellar activity baseline.

\section{MODELING THE PRE-TRANSIT ABSORPTION}

In Paper I, we modeled the pre-transit absorption as arising in a thin bow shock orbiting $\sim 13 R_{p}$ ahead of the planet. While this simple geometric model was able to account for the absorption time series and the strength of the measured absorption, the favored model parameters resulted in two relatively unlikely conclusions: 1 . A low stellar wind speed of $\sim 40 \mathrm{~km} \mathrm{~s}^{-1}$ at the planet's orbital radius; 2 . A very strong equatorial surface planetary magnetic field strength of $\sim 28 \mathrm{G}$. The low stellar wind speed is at odds with most MHD simulations of the slow solar and stellar winds (e.g., Cohen et al. 2007; Llama et al. 2013; Johnstone et al. 2015) which reach speeds of $\sim 200 \mathrm{~km} \mathrm{~s}^{-1}$ at $\sim 10 R_{*}$. We note, however, that observational constraints on the stellar winds of low-mass stars besides the Sun are weak. The large magnetic field strength from our model, derived assuming pressure balance between the incoming stellar wind and planetary magnetosphere, is $\sim 4$ times larger ${ }^{6}$ than that estimated by Reiners \& Christensen (2010) for HD 189733 b based on magnetic field strength scaling relations for brown dwarfs and giant planets. While field strengths of this magnitude are estimated for more massive planets, it is difficult to produce such a strong field for a Jupiter-mass object (Reiners et al. 2009; Reiners \& Christensen 2010).

The absorption time series shown in Figure 5 does not match the specific prediction of the bow shock model from Paper I. Furthermore, we were unsuccessful at finding a suitable bow shock model to describe the 2015 time series. As demonstrated in Section 4.1, the magnitude of the pre-transit absorption can be influenced by low level variations in the stellar activity level. If the observed signal is assumed to be solely associated with the planet, it is possible to explain the absorption as arising in transiting circumplanetary material. This motivated us to search for a geometry that is capable of reproducing the absorption.

\subsection{Clumpy Accretion}

One such geometry is an accretion stream escaping from the L1 point as the planet overflows its Roche lobe (Lai et al. 2010; Li et al. 2010). The relatively constant pre-transit $W_{\lambda}$ absorption values, the very early appearance of the absorption, and the duration of the pre-transit signals are suggestive of a clumpy accretion flow. This type of accretion flow, i.e., non-uniform and time variable spiraling in from the planet's orbit, is seen in specific 2D MHD simulations of (Matsakos et al. 2015 see also Bisikalo et al. (2013) for simulations of Roche lobe overflow where the accretion stream is halted by the stellar wind pressure).

We have modeled the pre-transit absorption as being caused by two distinct clumps of material that are spiraling in from the planet's orbital radius toward the central star. The choice of two clumps is motivated by the two distinct pre-transit absorption signatures seen in the $W_{\mathrm{H} \alpha}$ values in Figure 5. The spiral trajectory in this case is approximated as a straight line since the clumps are located relatively close to the planet. In fact, due to the combination of the Coriolis force and the gravitational attraction of the planet, the trajectory of the material near the

\footnotetext{
6 In Paper I, the magnetic field strength was given as $~ 2$ times stronger than the Reiners \& Christensen (2010) estimate. This was incorrect since the Reiners \& Christensen (2010) given value is $14 \mathrm{G}$ at the pole and not the equator. For a dipolar field, the equatorial value is half of the polar value.
} 
Table 2

Stellar, Planetary, and Accretion Clump Model Parameters

\begin{tabular}{lccc}
\hline \hline $\begin{array}{l}\text { Parameter } \\
(1)\end{array}$ & $\begin{array}{c}\text { Symbol } \\
(2)\end{array}$ & $\begin{array}{c}\text { Value }^{\mathrm{a}, \mathrm{b}} \\
(3)\end{array}$ & $\begin{array}{c}\text { Units } \\
(4)\end{array}$ \\
\hline Stellar radius & $R_{*}$ & 0.756 & $R_{\odot}$ \\
Stellar rotational velocity & $v \sin i$ & 3.10 & $\mathrm{~km} \mathrm{~s}^{-1}$ \\
Impact parameters & $b$ & 0.680 & $R_{*}$ \\
Orbital period & $P_{\text {orb }}$ & 2.218573 & days \\
Orbital velocity & $v_{\text {orb }}$ & 151.96 & $\mathrm{~km} \mathrm{~s}^{-1}$ \\
Orbital radius & $a$ & 0.03099 & $\mathrm{au}$ \\
Planetary radius & $R_{p}$ & 1.138 & $R_{J}$ \\
& & & \\
Time of clump initiation & $t_{\text {pre }}$ & $16.7,11.4$ & hours before mid- \\
& & & transit \\
Clump radius & $r_{c}$ & $0.5,0.5$ & $R_{p}$ \\
Clump length & $l_{c}$ & $1.0,0.5$ & $R_{p}$ \\
Clump density & $\rho_{c}$ & $1.5,4.0$ & $10^{-22} \mathrm{~g} \mathrm{~cm}^{-3}$ \\
Line broadening & $b_{c}$ & $5.7,5.7$ & $\mathrm{~km} \mathrm{~s}^{-1}$ \\
\multicolumn{1}{l}{ parameter } & & & \\
\hline
\end{tabular}

Notes.

${ }^{\mathrm{a}}$ With the exception of $v \sin i$, all stellar and planetary parameters taken from Torres et al. (2008). The $v \sin i$ value is taken from Collier Cameron et al. (2010).

${ }^{\mathrm{b}}$ Clump parameters listed for clump 1 and then clump 2.

L1 point can be approximated by a straight line (Lubow \& Shu 1975; Lai et al. 2010). Any deviations from a straight line trajectory are secondary effects on the transit light curve. Each clump is released from rest from the L1 point at $4.25 R_{p}$ at some time $t_{\text {pre }}$. The clump is then accelerated along a straight line trajectory that forms an angle $\theta_{c}$ with the planet's orbit. The angle $\theta_{c}$ is determined by the system mass ratio and in this case is $\sim 60^{\circ}$. The acceleration of the clump is $a_{c}=$ $0.0019 \mathrm{~km} \mathrm{~s}^{-2}=2.3 \times 10^{-8} R_{p} \mathrm{~s}^{-2}$, which is approximated from the $q=M_{p} / M_{*}=0.001$ case $(q=0.0013$ for the HD 189733 system) presented in Lai et al. (2010). For simplicity, we choose a cylindrical geometry for each clump defined by a radius $r_{c}$ and length $l_{c}$. The density of each clump is uniform. The line profiles of the transiting clump are calculated identically to the bow shock profiles in Paper I. The optical depth at line center for each grid point is

$$
\tau_{0}=\frac{\sqrt{\pi} e^{2} f_{l u} N_{l} \lambda_{l u}}{m_{e} c b},
$$

where $f_{l u}$ is the oscillator strength of the transition, $\lambda_{l u}$ is the central wavelength, $m_{e}$ is the electron mass, $e$ is the electron charge, $c$ is the speed of light in vacuum, and $N_{l}$ is the column density of the lower energy level of the transition. The line profile is a Doppler-broadened delta function, $\tau_{v}=\tau_{0} e^{-(v / b)^{2}}$, where $b=\sqrt{2} \sigma_{v}$ and $\sigma_{v}$ is the dispersion of a 1D Gaussian velocity distribution (Draine 2011). The line broadening parameter for the clumps, used to approximate the profile shape according to Equation (5), is $b=5.7 \mathrm{~km} \mathrm{~s}^{-1}$.

\subsection{In-transit Absorption}

In order to maintain focus on the pre-transit signal, we will present a detailed discussion of the in-transit data, including an analysis of velocities in the planetary atmosphere, in a subsequent paper. A brief summary of the in-transit model, also shown over-plotted in Figure 5, is given here. We model

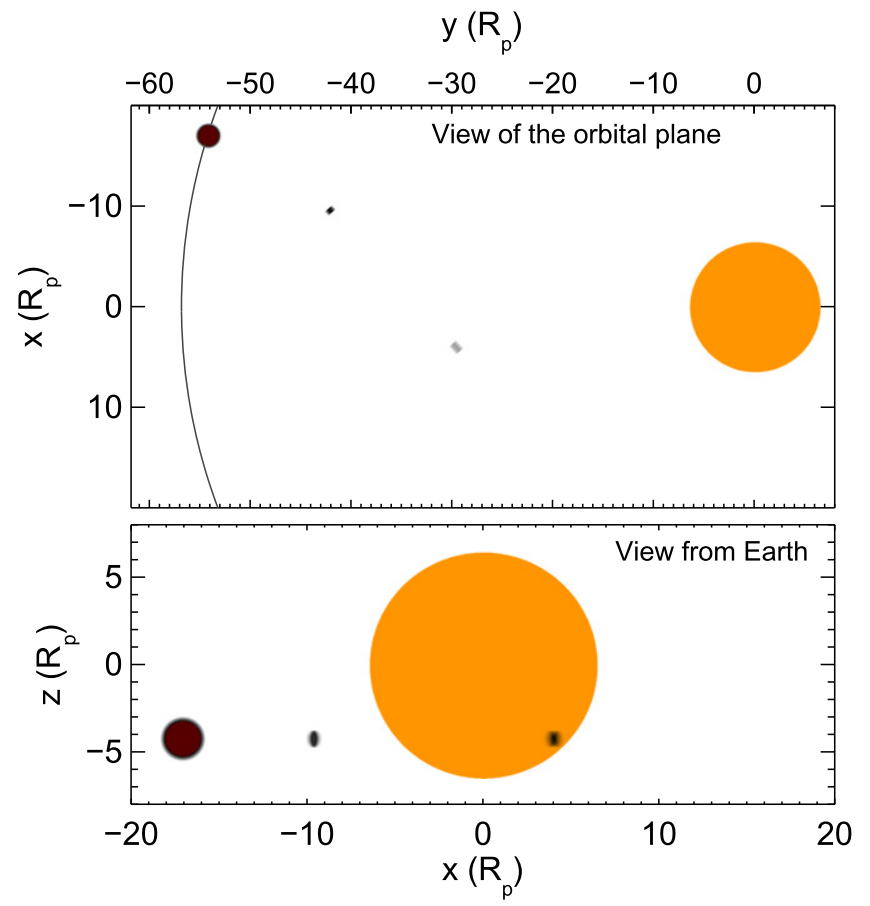

Figure 15. Views of the first transiting clump in the plane of the orbit (top panel) and perpendicular to the orbital plane (bottom panel) for the snapshot at $t-t_{\text {mid }}=-158$ minutes. The densities are scaled to the planetary atmosphere. The clumps represent stochastic mass loss events from the planet that are dense and extended enough to cause significant absorption in the cores of the Balmer lines.

the 2015 in-transit absorption as arising in an extended uniform density atmosphere. The choice of uniform density is motivated by the results of Christie et al. (2013) who find that the density of the dominant $n=2$ state (the $2 \mathrm{~s}$ state) remains fairly constant over almost three orders of magnitude in pressure $\left(10^{-6}-10^{-9}\right.$ bar $)$. This can be understood as the result of outwardly increasing temperatures offsetting the decreasing neutral hydrogen abundance. We assign a temperature to the base of the atmosphere and this sets the scale height, $H_{\mathrm{atm}}$. The $3 \mathrm{D}$ density grid is filled with material out to $10 H_{\mathrm{atm}}$ and the column density is calculated for each atmospheric point that occults the star. We find a good approximation of the average in-transit Balmer line profile shape and absorption values with a density $\rho=4.0 \times 10^{-23} \mathrm{~g} \mathrm{~cm}^{-3}$ and $T=6000 \mathrm{~K}$, which translates to a scale height of $H_{\text {exo }}=0.028 R_{p}$.

\subsection{Model Results}

When finding a model that roughly describes the pre-transit signal, we need to consider the strength and shape of the $W_{\lambda}$ time series, the ratios of the Balmer line $W_{\lambda}$ values, and the shape of the observed line profiles. While these constraints taken together significantly narrow the available parameter space, degeneracies exist between the model parameters. For example, increasing the length of the clump by $50 \%$ while decreasing the density by a factor of $\sim 3$ results in similar average $W_{\lambda}$ values. The increase in clump length, however, lengthens the egress and ingress time of the pre-transit features, which appear to be very brief ( $\sim 10$ minutes). In addition, the decreased density produces a narrower line profile as opacity broadening becomes less prominent. This results in less acceptable line profile matches between the data and the 
model. The final clump model values in Table 2 reflect our attempt to balance these constraints.

The model $W_{\lambda}$ values are shown as solid lines in Figure 5. The final clump parameters and the systems parameters used in the model are listed in Table 2. A view of the clump transit is shown in Figure 15 for $t-t_{\text {mid }}=-158$ minutes. $^{7}$ The clump model is successful at describing both the timing of the pretransit events and their absorption levels. We note that if the clumps are not allowed to accelerate, the transit duration is too long and the transit timing is significantly off. The densities of the clumps also roughly reproduce the Balmer line absorption ratios and line shapes, although most of the individual $\mathrm{H} \beta$ and $\mathrm{H} \gamma$ points are not measured at a significant level.

One piece of evidence that could support the accretion clump interpretation is the large $S_{\mathrm{HK}}$ value measured for the 2015 transit. If the star is in an active state, the planet will be subjected to higher levels of high-energy stellar radiation, increasing the mass loss rate (e.g., Murray-Clay et al. 2009; Trammell et al. 2011; Owen \& Adams 2014, 2016; Valsecchi et al. 2015). If enough material is evaporated from the atmosphere, the planet's magnetosphere can limit the outflow rate if the magnetic pressure dominates the gas pressure, creating a "dead zone" that extends many planetary radii Trammell et al. (2011, 2014), Khodachenko et al. (2016). Periodic mass loss, i.e., the accretion clumps, is not implausible from a filled magnetosphere that is being subjected to variable heating rates (e.g., Pillitteri et al. 2015). Furthermore, mass loss from HD $189733 \mathrm{~b}$ is observed to be variable (Lecavelier des Etangs et al. 2012). Although we do not explore the in-transit signal in detail here, the in-transit absorption appears to begin $\sim 15$ minutes before $t_{\mathrm{I}}$ and last at least 30 minutes after $t_{\mathrm{IV}}$. The planet moves across the star at $\sim 0.11 R_{p}$ minute $^{-1}$ which means the feature causing the absorption immediately pre- and posttransit extends $\sim 1.5 R_{p}$ ahead of the planet and $\sim 3.0 R_{p}$ behind the planet. This extended cloud of material is suggestive of a partially filled Roche lobe.

\subsection{Limitations to the Clumpy Accretion Interpretation}

While the accretion clump model can reproduce the observed absorption levels, and in addition to the parameter degeneracies discussed above, there are serious limitations to this interpretation. The first is the lack of large redshifted velocities in the pre-transit $\mathrm{H} \alpha$ transmission spectra (Figures 16 and 8). By the time the clumps transit the star, they are moving with line-ofsight velocities of $\sim 100$ and $\sim 70 \mathrm{~km} \mathrm{~s}^{-1}$, much larger than the $5-10 \mathrm{~km} \mathrm{~s}^{-1}$ line centroids seen for the individual spectra. This is the result of the large angle $\left(60^{\circ}\right)$ that the clump trajectory makes with the planet's orbit. This angle would need to be $\sim 5-10^{\circ}$ in order to produce redshifted velocities of $5-10 \mathrm{~km} \mathrm{~s}^{-1}$. A possible resolution to this problem is if the trajectory of the accreting material is altered by interactions with the stellar wind (e.g., see Matsakos et al. 2015), although modeling these interactions is beyond the scope of this paper. It is also not clear if simple hydrodynamic Roche-lobe overflow, as we've assumed here, is directly applicable in the hot Jupiter regime, especially when the confining effect of the planet's magnetosphere is taken into account (Trammell et al. 2011; Owen \& Adams 2014). Thus a more realistic treatment of the mass loss could allow the trajectory of the clumps to be less steep.

\footnotetext{
7 An animation of the clump transit is available at pwcastro.com/research/.
}

Finally, there is currently little theoretical evidence to support the existence of large $n=2$ neutral hydrogen populations at such short distances from the star. During transit, the clumps are within $\sim 5-6 R_{*}$ of the star, about $30 \%-$ $40 \%$ closer than the planet's orbit, and receive a factor of $\sim 2 \times$ more stellar radiation. Again, the clump trajectory could be less extreme through interactions with the stellar wind. Almost all of the escaping planetary material is photo-ionized by $\sim 5 R_{p}$ from the planet (e.g., Murray-Clay et al. 2009; Tripathi et al. 2015; Salz et al. 2016) and the stellar wind is entirely composed of ions. Thus some highly non-equilibrium process is needed to maintain a population of excited neutral hydrogen for at least the duration of the transit. It is not clear if such a process (e.g., time-dependent recombination or chargeexchange of stellar wind protons with the small population of planetary neutrals) is capable of producing the necessary neutral hydrogen density. Modeling of the interaction between the planetary accretion flow and the stellar wind and how this interaction affects the excited neutral hydrogen density would be useful to clarify this outstanding question.

\section{SUMMARY AND CONCLUSIONS}

We have presented new transit observations of HD 189733 b which cover a significant amount of pre-transit phase and a small amount of post-transit phase. Our results can be summarized as the following.

1. We detect strong in-transit absorption from the planetary atmosphere in $\mathrm{H} \alpha, \mathrm{H} \beta$, and $\mathrm{H} \gamma$. We also detect weak Na I $5896 \mathrm{~A}$ absorption and measure a marginal amount of Mg I $5184 \AA$ A absorption. An upcoming paper will present an interpretation of the in-transit $\mathrm{H} \alpha$ line centroid velocities.

2. We confirm the presence of a pre-transit Balmer line signature at a significant level for the 2015 data. However, the pre-transit signal observed in the 2015 transit differs significantly from that observed in Paper I. In particular, the 2015 pre-transit signal does not match the prediction of the specific bow shock model presented in Paper I.

3. We have reanalyzed the $\mathrm{Ca}$ II $\mathrm{H}$ line from the 2013 transit and found a moderate correlation between the $W_{\mathrm{H} \alpha}$ values and residual core flux of the $\mathrm{Ca}$ II lines. A weaker correlation was found between $W_{\mathrm{H} \alpha}$ and $W_{\mathrm{Ca} \text { II }}$ for the 2015 data. We suggest that the residual core flux be used in future studies as an activity proxy for short timescales rather than the $S_{\mathrm{HK}}$ index.

4. Motivated by the $W_{\mathrm{H} \alpha}-W_{\mathrm{Ca} \text { II }}$ correlations, we explored the potential of changing activity levels to produce the pre-transit signature. We find that even small levels of intrinsic stellar variation in the line cores can reproduce the observed transmission spectra for both pre-transit measurements. It is not clear, however, if changes in the stellar activity level on these timescales are common.

5. We have modeled the pre-transit absorption as arising in a clumpy accretion flow. The clumpy accretion model is able to reproduce both the timing and magnitude of the pre-transit events. However, there are significant drawbacks to the clumpy accretion model, the foremost of which is the lack of large redshifted absorption velocities that should be produced by ballistically in-falling material. 
Although we have modeled the pre-transit signature as arising in absorbing circumplanetary material, we caution against a firm interpretation of the data. The magnitude of the pre-transit detections from both the 2013 and 2015 data can be explained by changing levels of stellar activity. Due to the lack of detailed information concerning short-term, low-level variations in the $\mathrm{H} \alpha$ core flux, no conclusive argument can be made concerning one interpretation over the other. Both physical scenarios should be investigated for other hot planet systems to give the current study context. We are currently pursuing a high-cadence $\mathrm{H} \alpha$ monitoring campaign of HD 189733 , at all phases of the planet's orbit, with the intention of determining the frequency of these low-level changes in the core of the Balmer lines.

Pre-transit signatures around hot planets may provide unique information concerning the dynamics of escaping planetary material and, potentially, the structure and strength of the planetary magnetosphere. Hot planets around less active stars than HD 189733 would make prime targets for future investigations into pre-transit signatures since the observed signal can be attributed to the planet with a higher probability.

We are grateful to the referee for comments that helped improve this manuscript. The data presented herein were obtained at the W.M. Keck Observatory from telescope time allocated to the National Aeronautics and Space Administration through the agency's scientific partnership with the California Institute of Technology and the University of California. This work was supported by a NASA Keck PI Data Award, administered by the NASA Exoplanet Science Institute. The Observatory was made possible by the generous financial support of the W.M. Keck Foundation. The authors wish to recognize and acknowledge the very significant cultural role and reverence that the summit of Mauna Kea has always had within the indigenous Hawaiian community. We are most fortunate to have the opportunity to conduct observations from this mountain. This work was completed with support from the National Science Foundation through Astronomy and Astrophysics Research Grant AST-1313268 (PI: S.R.). P.W.C. is grateful for useful exchanges with $\mathrm{T}$. Matsakos concerning the morphology and dynamics of hot Jupiter accretion flows. P.W.C. and S.R. acknowledge helpful conversations with
D. Christie and C. Huang regarding the interpretation of neutral hydrogen absorption in the extended planetary atmosphere.

\section{APPENDIX A INDIVIDUAL TRANSMISSION SPECTRA}

Transmission spectra for individual exposures are shown in Figures 16-20. The out-of-transit spectra are marked with green crosses; in-transit spectra are marked with a magenta star. The solid green dots in Figures 19 and 20 show the spectra binned by 20 pixels. The individual transmission spectra are the weighted average of the ratio between the spectrum of interest and all combinations $(N=255$ for the eight comparison spectra) of the comparison spectra used to construct the master comparison spectrum. Transmission spectra for the individual comparison spectra are constructed by comparing the exposure of interest to a master comparison spectrum that includes all of the other comparison spectra besides the spectrum of interest. This weighted average is performed to prevent individual comparison spectra from having a large influence on the individual transmission spectra.

Overall, the individual spectra are well defined for $\mathrm{H} \alpha$ and for the strong $\mathrm{H} \beta$ absorption. The individual $\mathrm{H} \gamma$ spectra, however, are very noisy and the absorption is not obvious except for the times $t-t_{\text {mid }}=-54,-48$, and -41 minutes. We note that the telescope experienced minor guiding errors at $\sim-180$ minutes (see Figure 1). On the blue chip, where $\mathrm{H} \gamma$ is located, the orders are closely spaced and any significant deviation of the stellar profile can result in small amounts of order overlap. For these exposures, the stellar profile in the spatial direction on the blue CCD is $10 \%-15 \%$ wider than it is for the other exposures, over which it is constant to within $\sim 1 \%-2 \%$. We believe this is what accounts for the sawtooth pattern seen in the the $\mathrm{H} \gamma$ spectra from $t-t_{m}=-180$ to -167 minutes. Thus the absorption values calculated from these spectra are highly suspect. The individual $\mathrm{Na}$ I line profile morphologies are visible in a few of the pre-transit and intransit spectra in Figure 19. However, large residuals in the line core obscure the true shape of the line in most cases. The absorption, if present, is too weak to be seen in all but a few of the $\mathrm{Mg}$ I profiles in Figure 20. 


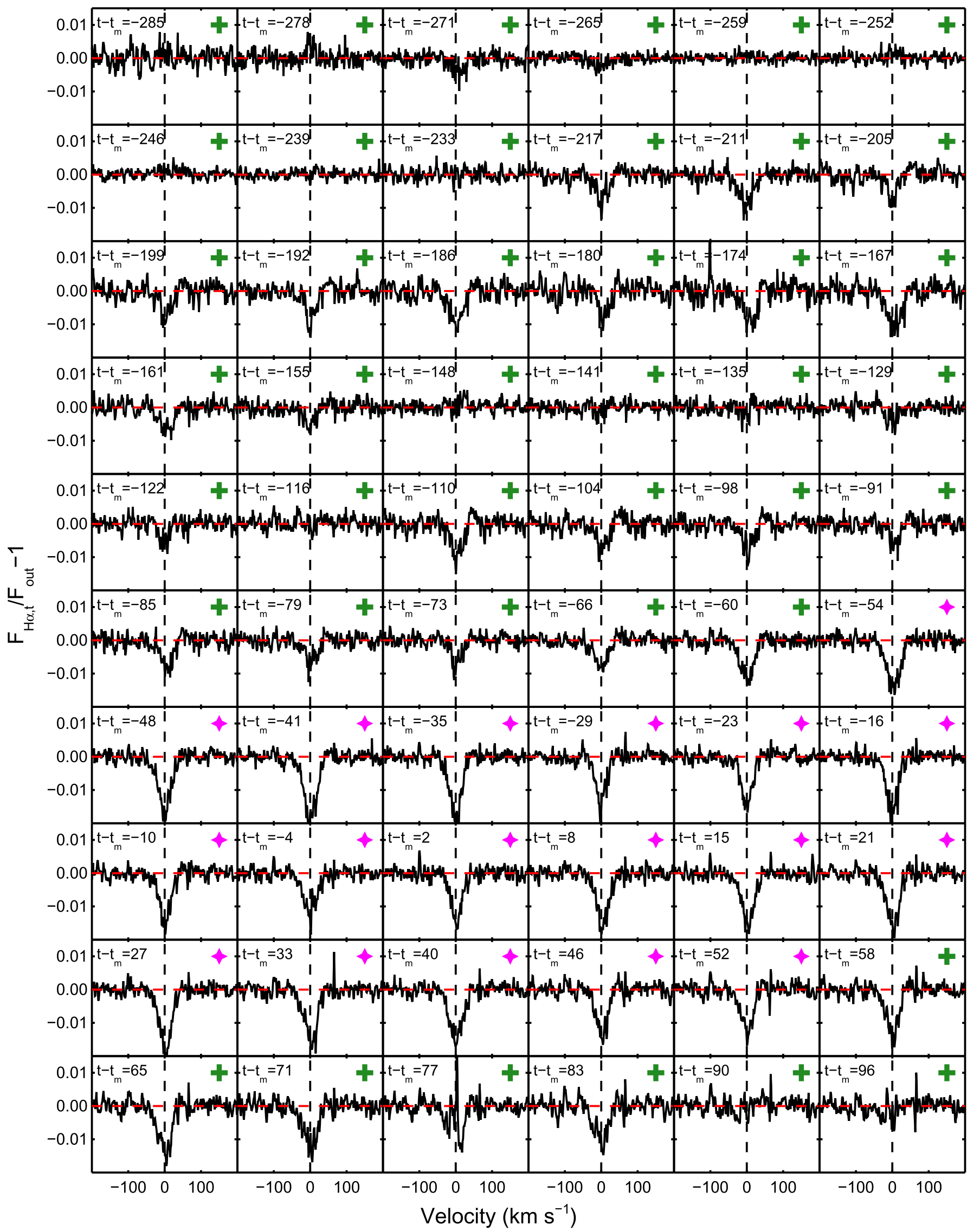

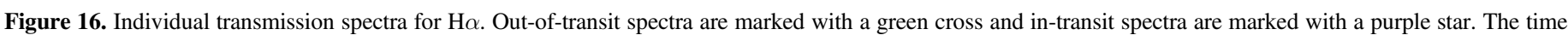
from mid transit in minutes is labeled $t-t_{m}$. 


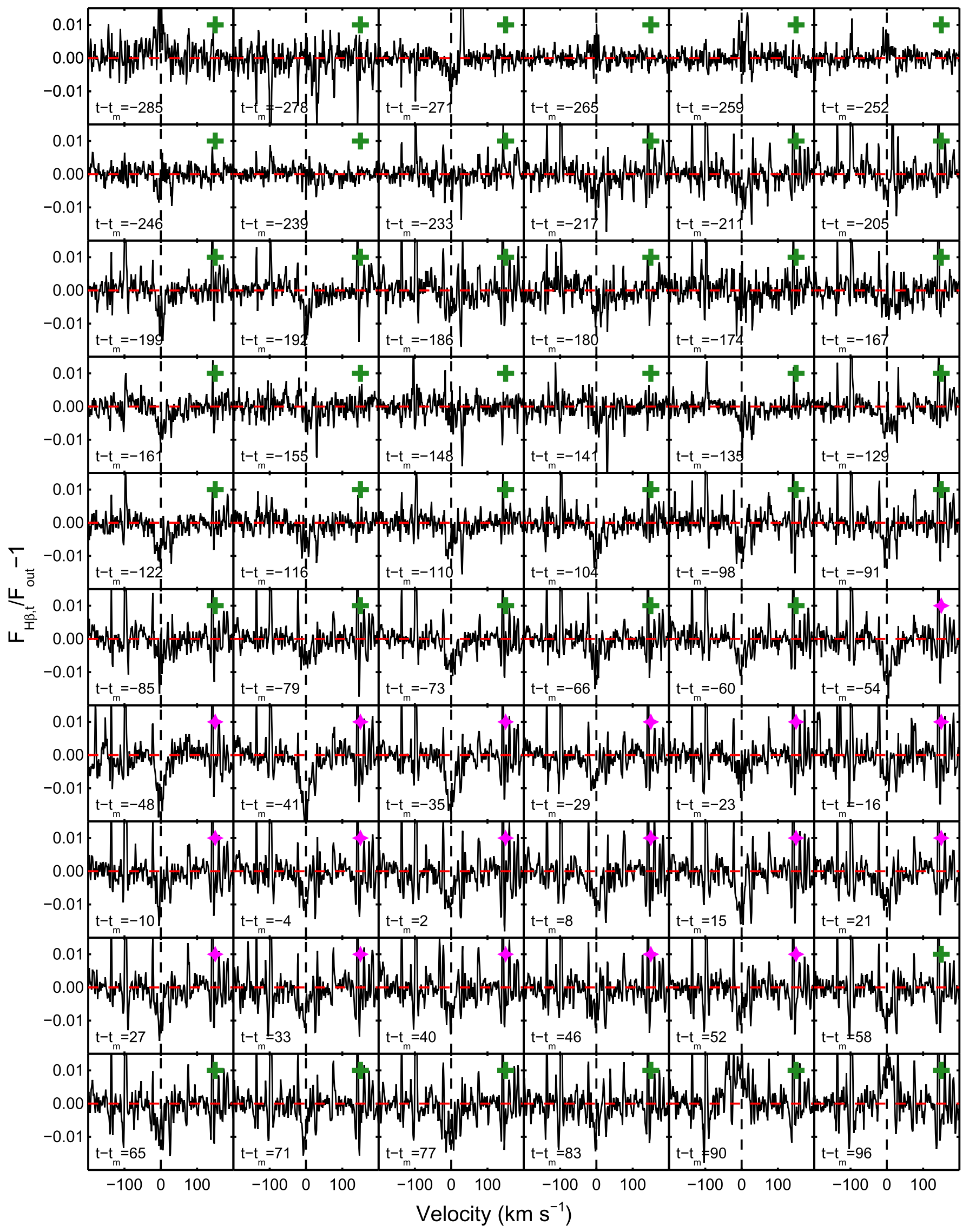

Figure 17. Individual transmission spectra for $\mathrm{H} \beta$. All markings are the same as Figure 16. 


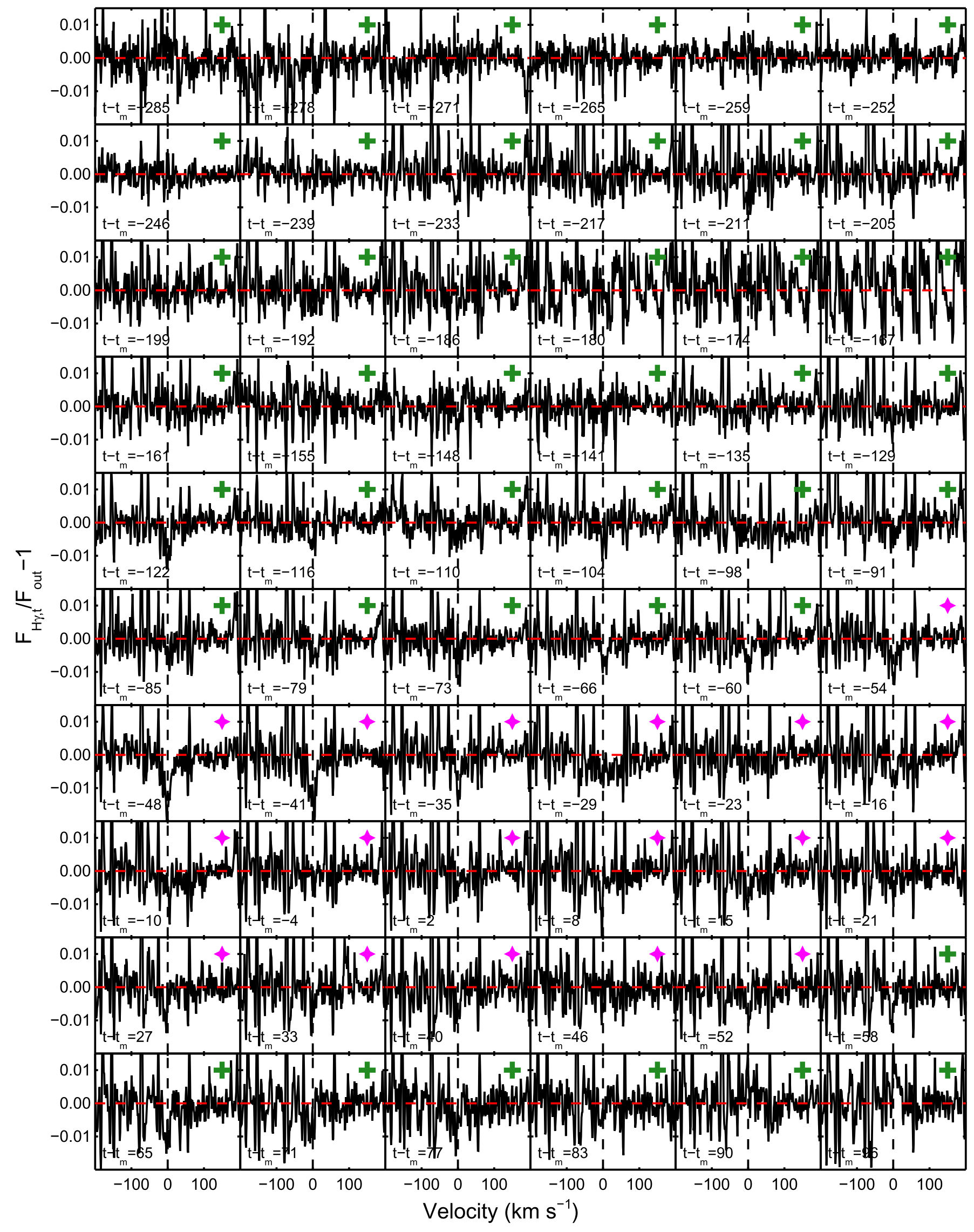

Figure 18. Individual transmission spectra for $\mathrm{H} \gamma$. All markings are the same as Figures 16 and 17 . The jagged sawtooth pattern in the spectra from $t-t_{m}=-180$ to -167 minutes is likely caused by poor guiding and small amounts of order overlap on the blue chip. 


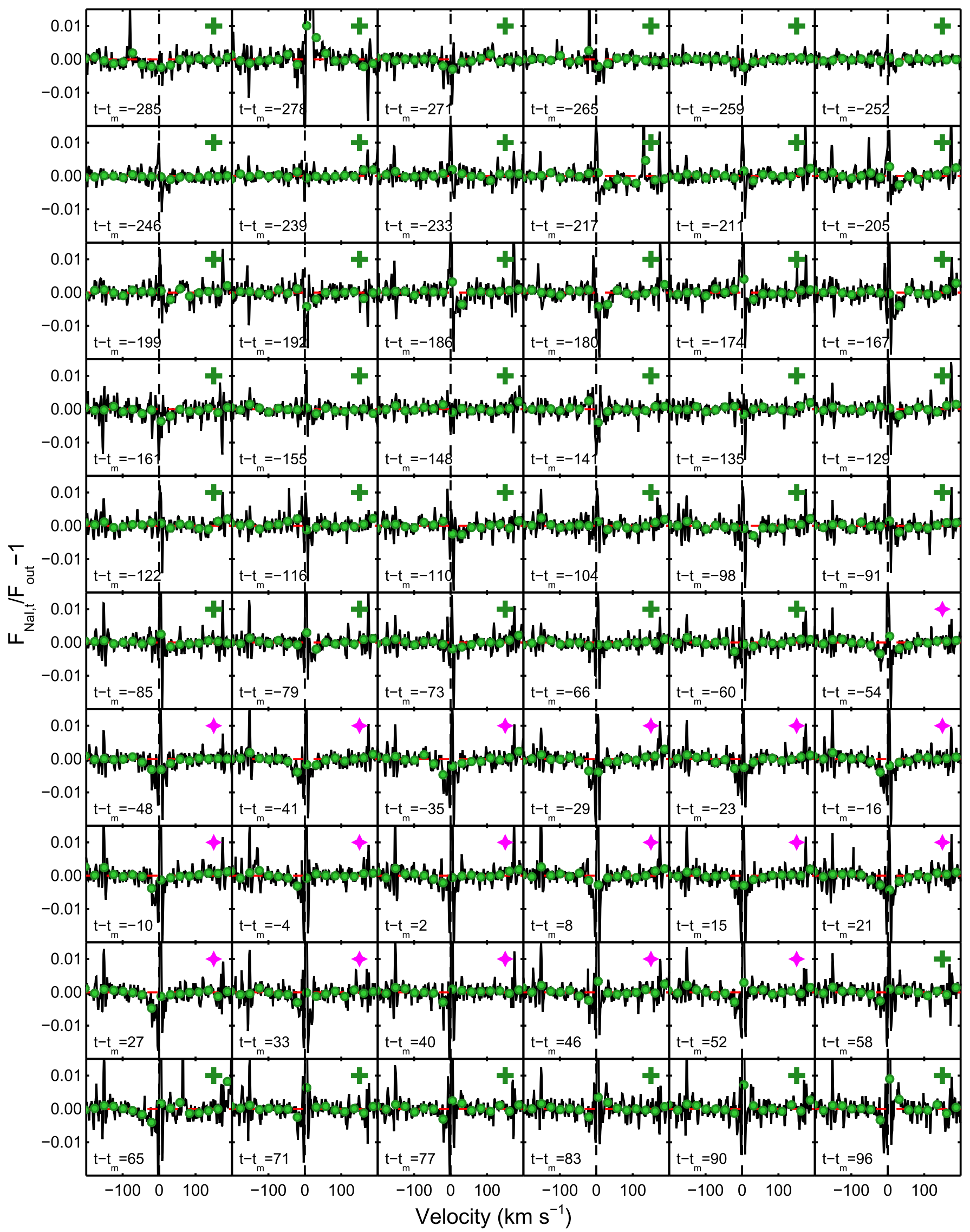

Figure 19. Individual transmission spectra for Na I $5896 \AA$. The green dots show the spectra binned by 20 pixels for clarity. Note the weak redshifted absorption feature in some of the pre-transit spectra. 


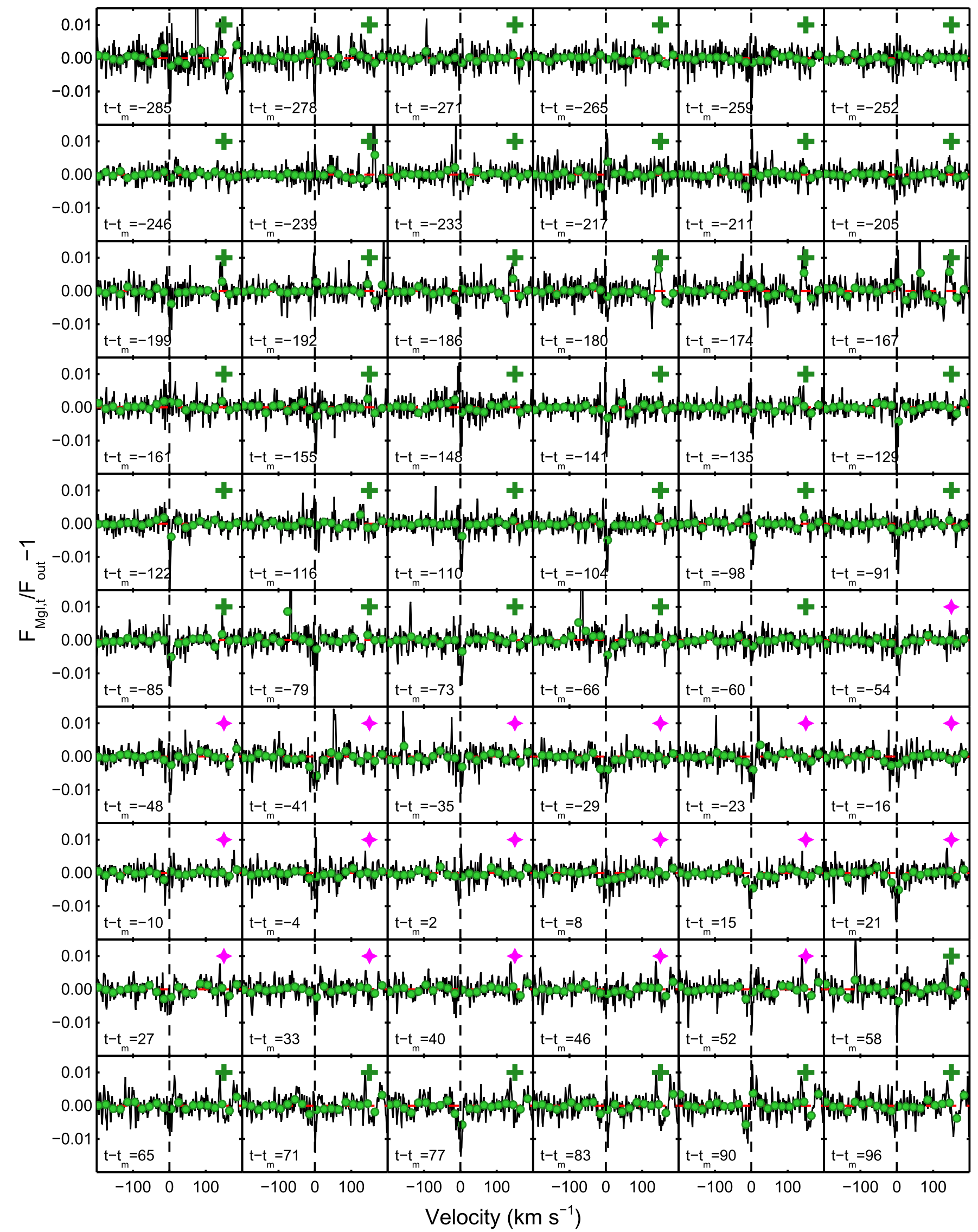

Figure 20. Individual transmission spectra for $\mathrm{Mg}_{\mathrm{I}} 5184 \AA$ A. The green dots show the spectra binned by 20 pixels for clarity. The in-transit absorption, if present, is very weak and not detected at a significant level. 
APPENDIX B

\section{CA II RESIDUAL CORE PROFILES}

This appendix contains the $\mathrm{Ca}$ II residual core profiles for both transit dates. Pre-transit profiles are shown in pink, in-transit profiles in purple, and post transit profile in blue.

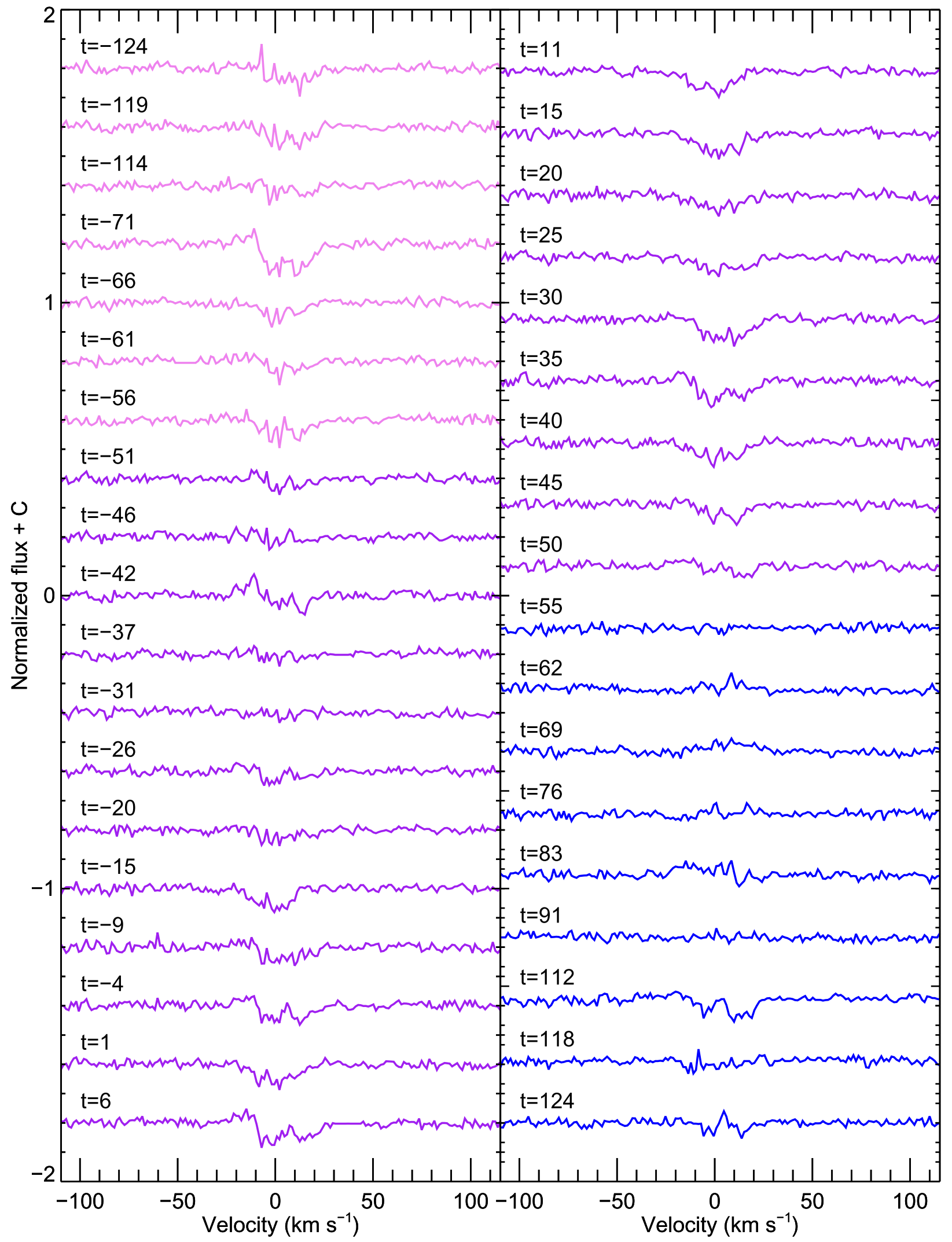

Figure 21. Residual $\mathrm{Ca}$ II $\mathrm{H}$ core profiles for the 2013 transit. The time given for each observation is in units of minutes from mid-transit. 


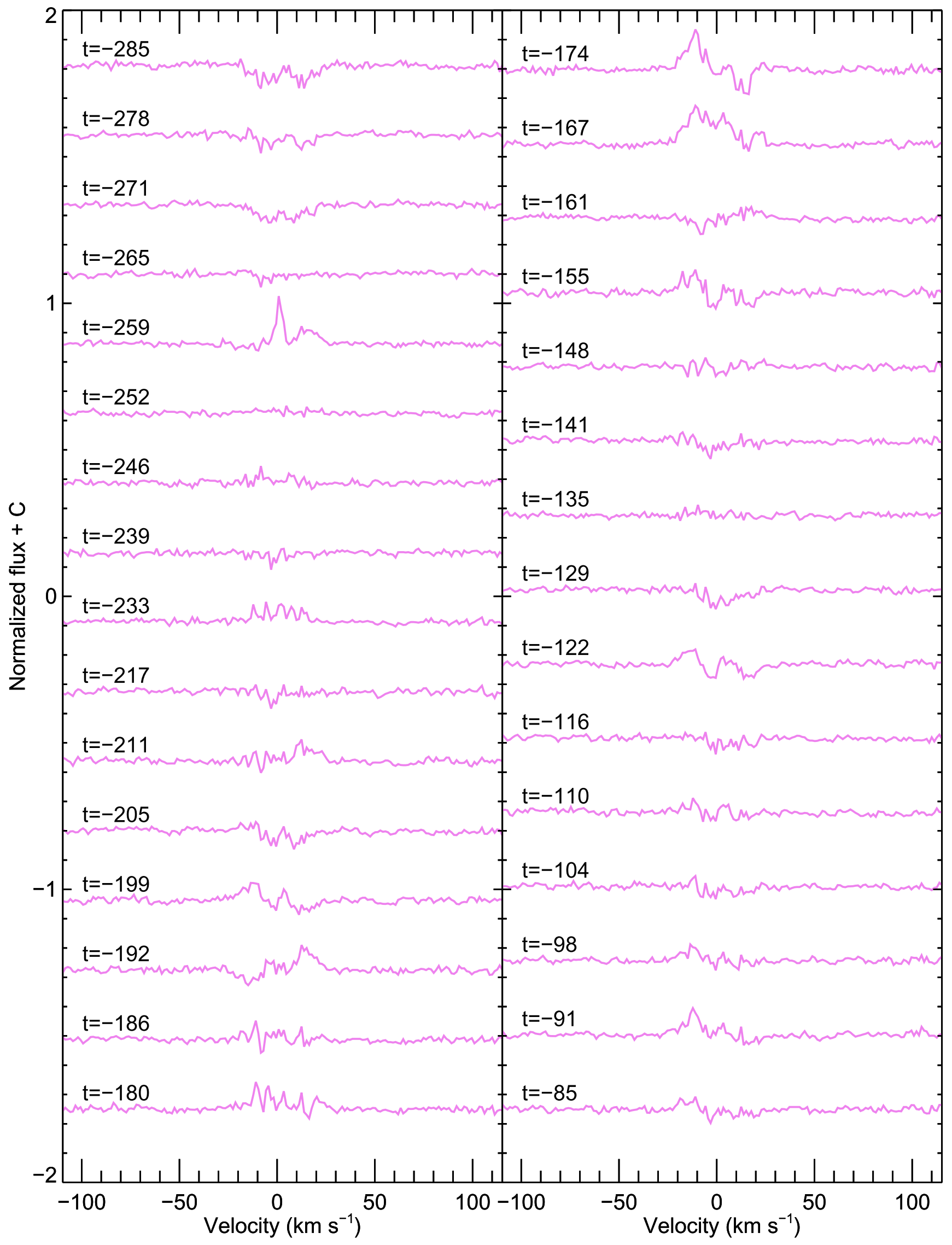

Figure 22. Residual Ca II H core profiles for the first part of the 2015 transit. Colors and labels are the same as Figure 21. 


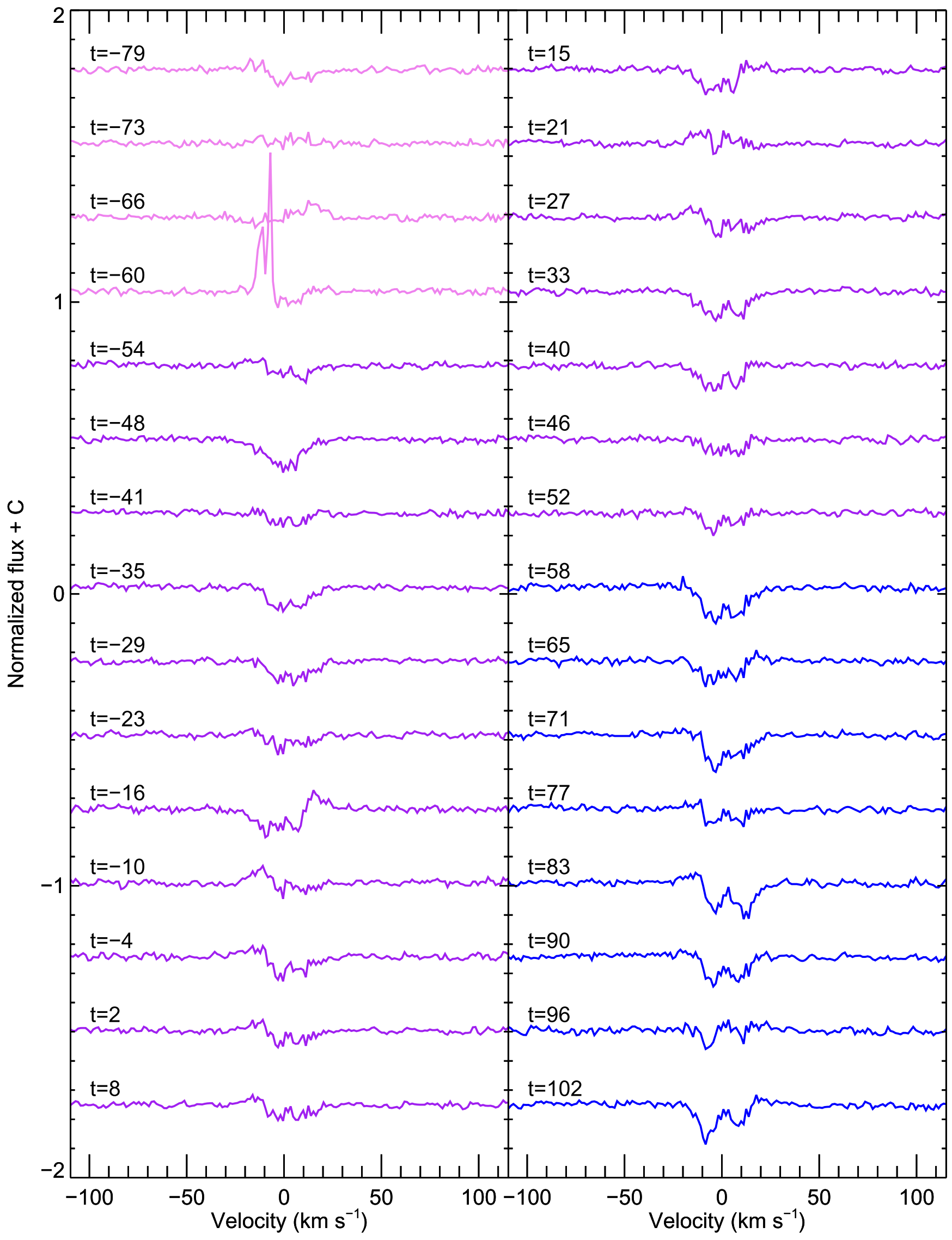

Figure 23. Residual Ca II H core profiles for the second part of the 2015 transit. Colors and labels are the same as Figures 21 and 22. 


\section{REFERENCES}

Andretta, V., \& Giampapa, M. S. 1995, ApJ, 439, 405

Ben-Jaffel, L., \& Ballester, G. E. 2013, A\&A, 553, A52

Bento, J., Wheatley, P. J., Copperwheat, C. M., et al. 2014, MNRAS, 437, 1511

Berta, Z. K., Charbonneau, D., Bean, J., et al. 2011, ApJ, 736, 12

Bisikalo, D., Kaygorodov, P., Ionov, D., et al. 2013, ApJ, 764, 19

Boisse, I., Moutou, C., Vidal-Madjar, A., et al. 2009, A\&A, 495, 959

Bouchy, F., Udry, S., Mayor, M., et al. 2005, A\&A, 444, L15

Bourrier, V., Ehrenreich, D., \& Lecavelier des Etangs, A. 2015, A\&A, 582, A65

Bourrier, V., Lecavelier des Etangs, A., Dupuy, H., et al. 2013, A\&A, 551, A63

Bourrier, V., Lecavelier des Etangs, A., \& Vidal-Madjar, A. 2015, A\&A, 573, A11

Brogi, M., de Kok, R. J., Albrecht, S., et al. 2016, ApJ, 817, 106

Cauley, P. W., Redfield, S., Jensen, A. G., et al. 2015, ApJ, 810, 13

Christie, D., Arras, P., \& Li, Z.-Y. 2013, ApJ, 772, 144

Cincunegui, C., Díaz, R. F., \& Mauas, P. J. D. 2007, A\&A, 469, 309

Cohen, O., Drake, J. J., Kashyap, V. L., Sokolov, I. V., \& Gombosi, T. I. 2010, ApJL, 723, L64

Cohen, O., Kashyap, V. L., Drake, J. J., et al. 2011, ApJ, 733, 67

Cohen, O., Sokolov, I. V., Roussev, I. I., et al. 2007, ApJL, 654, L163

Collier Cameron, A., Bruce, V. A., Miller, G. R. M., Triaud, A. H. M. J., \& Queloz, D. 2010, MNRAS, 403, 151

Cuntz, M., Saar, S. H., \& Musielak, Z. E. 2000, ApJL, 533, L151

Czesla, S., Klocová, T., Khalafinejad, S., Wolter, U., \& Schmitt, J. H. M. M. 2015, A\&A, 582, A51

Di Gloria, E., Snellen, I. A. G., \& Albrecht, S. 2015, A\&A, 580, A84

Draine, B. T. 2011, Physics of the Interstellar and Intergalactic Medium (Princeton, NJ: Princeton Univ. Press)

Duncan, D. K., Vaughan, A. H., Wilson, O. C., et al. 1991, ApJS, 76, 383

Ehrenreich, D., Bourrier, V., Wheatley, P. J., et al. 2015, Natur, 522, 459

Fares, R., Donati, J.-F., Moutou, C., et al. 2010, MNRAS, 406, 409

Fossati, L., Haswell, C. A., Froning, C. S., et al. 2010, ApJL, 714, L222

Garcá-Alvarez, D., Johns-Krull, C. M., Doyle, J. G., et al. 2005, A\&A, 444, 593

Gomes da Silva, J., Santos, N. C., Boisse, I., Dumusque, X., \& Lovis, C. 2014, A\&A, 566, 66

Isaacson, H., \& Fischer, D. 2010, ApJ, 725, 875

Jensen, A. G., Redfield, S., Endl, M., et al. 2011, ApJ, 743, 203

Jensen, A. G., Redfield, S., Endl, M., et al. 2012, ApJ, 751, 86

Johns-Krull, C. M., Hawley, S. L., Basri, G., \& Valenti, J. A. 1997, ApJS, 112,221

Johnstone, C. P., Güdel, M., Lüftinger, T., Toth, G., \& Brott, I. 2015, A\&A, 577, A27

Kausch, W., Noll, S., Smette, A., et al. 2014, in ASP Conf. Ser. 485, Astronomical Data Analysis Software and Systems XXIII, ed. N. Manset, \& P. Forshay (San Francisco, CA: ASP), 403

Khodachenko, M. L., Shaikhislamov, I. F., Lammer, H., \& Prokopov, P. A. 2016, ApJ, 813, 50

Kowalski, A. F., Hawley, S. L., Wisniewski, J. P., et al. 2013, ApJS, 207, 15

Kuridze, D., Mathioudakis, M., Simões, P. J. A., et al. 2015, ApJ, 813, 125

Lai, D., Helling, Ch., \& van den Heuvel, E. P. J. 2010, ApJ, 721, 923

Lalitha, S., Fuhrmeister, B., Wolter, U., et al. 2013, A\&A, 560, A69

Lanza, A. F. 2010, A\&A, 512, A77

Lecavelier des Etangs, A., Bourrier, A., Wheatley, P. J., et al. 2012, A\&A, $543, \mathrm{~L} 4$
Lecavelier des Etangs, A., Ehrenreich, D., Vidal-Madjar, A., et al. 2010, A\&A, 514, A72

Li, S.-L., Miller, N., Lin, D. N. C., \& Fortney, J. J. 2010, Natur, 463, 1054

Livingston, W., Wallace, L., White, O. R., \& Giampapa, M. S. 2007, ApJ, 657,1137

Llama, J., Vidotto, A. A., Jardine, M., et al. 2013, MNRAS, 436, 2179

Llama, J., Wood, K., Jardine, M., et al. 2011, MNRAS, 416, L41

Lodders, K. 1999, ApJ, 519, 793

Lopez, E. D., \& Fortney, J. J. 2013, ApJ, 776, 2

Louden, T., \& Wheatley, P. J. 2015, ApJL, 814, L24

Lubow, S. H., \& Shu, F. H. 1975, ApJ, 198, 383

Matsakos, T., Uribe, A., \& Königl, A. 2015, A\&A, 578, A6

Menou, K., \& Rauscher, E. 2010, ApJ, 713, 1174

Meunier, N., \& Delfosse, X. 2009, A\&A, 501, 1103

Murphy, T., Bell, M. E., Kaplan, D. L., et al. 2015, MNRAS, 446, 2560

Murray-Clay, R. A., Chiang, E. I., \& Murray, N. 2009, ApJ, 693, 23

Owen, J., \& Adams, F. C. 2016, MNRAS, 456, 3053

Owen, J. E., \& Adams, F. C. 2014, MNRAS, 444, 3761

Owen, J. E., \& Jackson, A. P. 2012, MNRAS, 425, 2931

Pillitteri, I., Maggio, A., Micela, G., et al. 2015, ApJ, 805, 52

Rauscher, E., \& Kempton, E. M. R. 2014, ApJ, 790, 79

Redfield, S., Endl, M., Cochran, W. D., \& Koesterke, L. 2008, ApJL, 673, L87

Reiners, A., Basri, G., \& Christensen, U. R. 2009, ApJ, 697, 373

Reiners, A., \& Christensen, U. R. 2010, A\&A, 522, A13

Salz, M., Czesla, S., Schneider, P. C., \& Schmitt, J. H. M. M. 2016, A\&A, 586,75

Scandariato, G., Maggio, A., Lanza, A. F., et al. 2013, A\&A, 552, A7

Shapiro, A. I., Solanki, S. K., Krivova, N. A., et al. 2015, A\&A, 569, A38

Shkolnik, E., Bohlender, D. A., Walker, G. A. H., \& Collier Cameron, A. 2008 ApJ, 676, 628

Shkolnik, E., Walker, G. A. H., Bohlender, D. A., Gu, P.-G., \& Kürster, M. 2005, ApJ, 622, 1075

Showman, A., \& Guillot, A. P. 2002, A\&A, 385, 166

Snellen, I. A. G., Brandl, B. R., de Kok, R. J., et al. 2014, Natur, 509, 63

Strugarek, A., Brun, A. S., Matt, S. P., \& Réville, V. 2014, ApJ, 795, 86

Torres, G., Winn, J. N., \& Holman, M. J. 2008, ApJ, 677, 1324

Trammell, G. B., Arras, P., \& Li, Z.-Y. 2011, ApJ, 728, 152

Trammell, G. B., Zhi-Yun, L., \& Arras, P. 2014, ApJ, 788, 161

Tripathi, A., Kratter, K. M., Murray-Clay, R. A., \& Krumholz, M. R. 2015, ApJ, 808, 173

Turner, J. D., Christie, D., Arras, P., Johnson, R. E., \& Schmidt, C. 2016, MNRAS, 458, 3880

Turner, J. D., Smart, B. M., Hardgree-Ullman, K. M., et al. 2013, MNRAS, 428,678

Valsecchi, F., Rappaport, S., Rasio, F. A., Marchant, P., \& Rogers, L. A. 2015, ApJ, 813, 101

Vidal-Madjar, A., Huitson, C. M., Bourrier, V., et al. 2013, A\&A, 560, 54

Vidal-Madjar, A., Lecavelier des Etangs, A., Désert, J.-M., et al. 2003, Natur, 422, 143

Vidotto, A. A., Fares, R., Jardine, M., Moutou, C., \& Donati, J.-F. 2015, MNRAS, 449, 4117

Vidotto, A. A., Jardine, M., \& Helling, Ch. 2010, ApJ, 722L, 168

Vidotto, A. A., Jardine, M., \& Helling, Ch. 2011, MNRAS, 414, 1573

Vogt, S. S., Allen, S. L., Bigelow, B. C., et al. 1994, Proc. SPIE, 2198, 362

Winn, J. N., Holman, M. J., Henry, G. W., et al. 2007, AJ, 133, 1828

Wright, J. T., Marcy, G. W., Butler, R. P., \& Vogt, S. S. 2004, ApJS, 152, 261

Wyttenbach, A., Ehrenreich, D., Lovis, C., Udry, S., \& Pepe, F. 2015, A\&A, 577, A62

Zellem, R. T., Griffith, C. A., Pearson, K. A., et al. 2015, ApJ, 810, 11 